\title{
Polar Actions on Hermitian and Quaternion-Kähler Symmetric Spaces
}

\author{
Inaugural-Dissertation \\ zur \\ Erlangung des Doktorgrades \\ der Mathematisch-Naturwissenschaftlichen Fakultät \\ der Universität zu Köln
}

vorgelegt von

Samuel Tebege

aus Addis Abeba

Hundt-Druck GmbH, Köln 


\begin{abstract}
We analyze polar actions on Hermitian and quaternion-Kähler symmetric spaces. For complex integrable polar actions on Hermitian symmetric spaces of compact type we prove a reduction theorem and several corollaries concerning the geometry of these actions. The results are independent of the classification of polar actions on Hermitian symmetric spaces. In the second part we prove that polar actions on Wolf spaces are quaternion-coisotropic and that isometric actions on these spaces admit an orbit of special type, analogous to the existence of a complex orbit for an isometric action on a compact homogeneous simply-connected Kähler manifold.
\end{abstract}





\section{Contents}

Zusammenfassung $\quad$ i

Introduction $\quad$ v

1 Polar actions 1

1.1 Introduction and examples . . . . . . . . . . . . . . . 1

1.2 Basic properties . . . . . . . . . . . . . . 2

2 Polar actions on Hermitian symmetric spaces 5

2.1 Introduction . . . . . . . . . . . . . . . 5

2.1.1 Hermitian symmetric spaces as generalized flag manifolds 5

2.1 .2 Torus actions and toric varieties . . . . . . . . 6

2.1.3 The symplectic geometry of Hermitian symmetric spaces . 8

2.2 The Reduction Theorem . . . . . . . . . . . . . . . . . . . . . 11

2.3 Applications of the Reduction Theorem . . . . . . . . . . . 18

2.3.1 A hyperpolarity criterion . . . . . . . . . . 18

2.3.2 The rank one case . . . . . . . . . . . . . . . 19

2.3.3 Hermitian symmetric spaces of maximal rank . . . . . . . 20

2.3.4 Two examples: $\operatorname{Sp}(2) / \mathrm{U}(2)$ and $\operatorname{Sp}(3) / \mathrm{U}(3) \ldots \ldots . . .23$

3 Polar actions on Wolf spaces $\quad 25$

3.1 Quaternion-Kähler manifolds . . . . . . . . . . . . 25

3.2 Polar actions on Wolf spaces . . . . . . . . . . . . 28

$\begin{array}{ll}\text { Appendix } & 33\end{array}$

$\begin{array}{ll}\text { Bibliography } & 37\end{array}$ 


\section{Zusammenfassung}

Die systematische Untersuchung polarer Wirkungen begann mit $A$ general theory of canonical forms durch Palais und Terng [PaTe 1987]. Der Spezialfall der hyperpolaren Wirkungen wurde schon wesentlich früher betrachtet. Conlon zum Beispiel bewies, dass hyperpolare Wirkungen (er nannte sie actions admitting a K-transversal domain) variationell vollständig sind [Co 1971]. Dies verallgemeinerte Ergebnisse von Bott und Samelson, die gezeigt hatten, dass die adjungierte Wirkung einer kompakten Liegruppe und die Isotropiedarstellung eines kompakten symmetrischen Raumes ebenfalls variationell vollständig sind [BoSa 1958].

Polare Darstellungen wurden von Dadok klassifiziert und als Folge dieser Klassifikation stellte sich heraus, dass polare Darstellung Orbit-äquivalent zu $s$-Darstellungen sind [Dad 1985]. Kombiniert man dies mit der Tatsache, dass die Scheibendarstellung einer beliebigen polaren Wirkung wieder polar ist, zeigt dies, dass die Geometrie polarer Wirkungen eng mit der symmetrischer Räume verwandt ist. Tatsächlich bilden polare Wirkungen auf symmetrischen Räumen auch eine sehr große Beispielklasse solcher Wirkungen. Es fiel schnell auf, dass alle bekannten Beispiele polarer Wirkungen auf kompakten symmetrischen Räumen von höherem Rang schon hyperpolar sind. Die Frage, ob dies immer der Fall sein muss, ist noch nicht vollständig geklärt. Allerdings gibt es Antworten für eine große Klasse von Räumen, die ersten für die Klasse der Hermitesch-symmetrischen Räume. Podestà und Thorbergsson klassifizierten polare Wirkungen auf der komplexen Quadrik, indem sie ihr Ergebnis benutzten, dass polare Wirkungen auf diesen Räumen koisotrop sind [PoTh 2002]. Derselbe Ansatz wurde von Gori und Biliotti auf die komplexen Grassmannschen [BiGo 2005] und schließlich von Biliotti auf die restlichen Hermiteschsymmetrischen Räume angewandt [Bi 2006]. Es stellte sich heraus, dass polare Wirkungen auf kompakten, irreduziblen Hermitesch-symmetrischen Räumen von höherem Rang hyperpolar sind.

Kollross schloss vor kurzem die Klassifikation polarer Wirkungen auf kompakten symmetrischen Räumen höheren Ranges vom Typ I ab und zeigte so, dass auf diesen Räumen polare Wirkungen schon hyperpolar sind [Kol 2006]. Prinzipiell funktioniert seine Methode auch für symmetrische Räume vom Typ II, allerdings wird hier der rechnerische Aufwand recht groß.

Der erste Teil dieser Arbeit beschäftigt sich mit polaren Wirkungen auf Hermitesch-symmetrischen Räumen. Ziel ist es, geometrische Methoden zu entwickeln, die bei einem konzeptionellem Beweis des oben beschriebenen Phänomens hilfreich sein können. Insbesondere sind unsere Ergebnisse unabhängig 
von den erwähnten Klassifikationsergebnissen. Als besonders hilfreich erweist sich die Existenz einer Momentumabbildung für isometrische Wirkungen auf diesen Räumen. Das Hauptergebnis des ersten Teiles ist ein Reduktionstheorem, das sowohl die wirkende Gruppe auf einen Torus als auch die Kodimension des Schnittes reduziert. Es sei also $M$ ein irreduzibler Hermitsch-symmetrischer Raum vom kompakten Typ und $H$ eine kompakte, zusammenhängende Liegruppe, die polar und komplex-integrabel auf $M$ wirkt. Ferner sei $\mu: M \rightarrow \mathfrak{h}^{*}$ eine Momentumabbildung für die $H$-Wirkung.

Theorem. Es sei $p \in M$ ein regulärer Punkt der $H$-Wirkung, $\Sigma$ der Schnitt durch $p$ und $T:=H_{\mu(p)} / H_{p}$. Dann gilt:

(i) $\Sigma^{\mathbb{C}}:=\overline{T^{\mathbb{C}} \cdot p}$ ist eine totalgeodätische Untermannigfaltigkeit von $M$, die $\Sigma$ als eine Lagrangesche Untermannigfaltigkeit enthält.

(ii) Die T-Wirkung auf $\Sigma^{\mathbb{C}}$ ist wieder polar mit $\Sigma$ als Schnitt.

(iii) $\Sigma^{\mathbb{C}}$ spaltet in ein Produkt komplex-projektiver Räume.

Dieses Theorem hat mehrere Konsequenzen.

Korollar. Die H-Wirkung ist genau dann hyperpolar wenn es eine totalgeodätische Momentumfaser durch einen regulären Punkt gibt.

Das Reduktionstheorem erlaubt auch Aussagen über den Schnitt selbst:

Korollar. Die Schnitte der H-Wirkung sind Produkte von reell-projektiven Räumen.

Zusammen mit der Beobachtung, dass jede polare Wirkung auf komplexprojektiven Räumen komplex-integrabel ist, folgt:

Korollar. Es sei X ein komplex-projektiver Raum und H eine kompakte zusammenhängende Liegruppe, die polar auf $X$ wirkt. Dann ist die Wirkung komplexintegrabel und die Schnitte sind reell-projektive Räume.

Beispiele komplex-integrabler polarer Wirkungen sind durch polare $\mathbb{C}$-asystatische gegeben:

Proposition. Es sei $M$ eine irreduzible, kompakte, einfach-zusammenhängende, homogene Kählermannigfaltigkeit. Dann ist jede polare $\mathbb{C}$-asystatische Wirkung einer kompakten, zusammenhängenden Liegruppe komplex-integrabel.

Für ein weiteres Verständnis polarer Wirkungen ist es unerlässlich, die Wirkungen des Torus' $T=H_{\mu(p)} / H_{p}$ und dessen Komplexifizierung $T^{\mathbb{C}} \mathrm{zu}$ verstehen. Wie verhalten sich der Punkt $p$ und der Torus $T=H_{\mu(p)} / H_{p}$ zueinander? Die Theorie algebraischer Toruswirkungen auf verallgemeinerten Flaggenvarietäten ist besonders gut entwickelt für Tori maximalen Ranges. Dies erlaubt es uns für die Serie $C I(M=\mathrm{Sp}(n) / \mathrm{U}(n))$ weitere Aussagen zu treffen. Wir benutzen hier, dass dies Räume maximalen Ranges sind, was bedeutet, dass rk $G / K=$ rk $G$. Als erstes identifizieren wir mittels Ergebnissen von Dabrowski [Da 1996] den generischen Torusorbit-Typ von $M$. 
Proposition. Es sei $M=G / K=\operatorname{Sp}(n) / \mathrm{U}(n)$ und $T^{\mathbb{C}} \subset G^{\mathbb{C}}$ ein maximaler Torus. Dann ist der Abschluss eines generischen $T^{\mathbb{C}}$-Orbits isomorph zu einem Produkt von n komplex-projektiven Geraden.

Hier ist zu beachten, dass dies gerade der Torusorbit-Typ für die Komplexifizierung des Torus' $T:=K_{\mu(p)} / K_{p}$ ist. Es folgt, dass für eine hyperpolare Wirkung der Punkt $p$ im nicht-degenerierten Stratum $Z\left(T^{\mathbb{C}}\right)$ enthalten ist. Dieses Stratum ist als der Schnitt über die dicken Zellen aller Borel-Untergruppen, die $T^{\mathbb{C}}$ enthalten, definiert.

Proposition. Es sei $H \times M \rightarrow M$ eine komplex-integrable hyperpolare Wirkung maximaler Kohomogenität einer kompakten, zusammenhängenden Liegruppe auf $M=\operatorname{Sp}(n) / \mathrm{U}(n)$. Dann ist jeder reguläre Punkt $p \in M$ im nicht-degenerierten Stratum $Z\left(T^{\mathbb{C}}\right)$ der Komplexifizierung des Torus' $T:=H_{\mu(p)} / H_{p}$ enthalten.

Wir beenden das Kapitel über Hermitsch-symmetrische Räume, indem wir diese Ergebnisse auf zwei niedrig-dimensionale Beispiele anwenden.

Proposition. (i) Jede komplex-integrable polare Wirkung einer kompakten, zusammenhängenden Liegruppe auf $M_{2}:=\mathrm{Sp}(2) / \mathrm{U}(2)$ ist hyperpolar.

(ii) Jede komplex-integrable polare Wirkung maximaler Kohomogenität einer kompakten, zusammenhängenden Liegruppe auf $M_{3}:=\mathrm{Sp}(3) / \mathrm{U}(3)$ ist hyperpolar.

Der letzte Teil dieser Arbeit beschäftigt sich mit polaren Wirkungen auf quaternion-Kähler symmetrischen Räumen vom kompakten Typ, den sogenannten Wolf-Räumen. Wir verallgemeinern zuerst das Hauptergebnis aus [PoTh 2002] auf diese Räume.

Theorem. Es sei $M$ ein Wolf-Raum und $H$ eine kompakte, zusammenhängende Liegruppe, die polar auf $M$ wirkt. Dann ist die H-Wirkung quaternionalkoisotrop.

Im Beweis fließt in essentieller Weise das Klassifikationsergebnis von Kollross ein [Kol 2006]. Eine der Hauptschwierigkeiten in der Verallgemeinerung des Beweises für den Kähler-Fall liegt darin, dass wir nicht die Existenz eines quaternionalen Orbits garantieren können. Wir zeigen aber:

Theorem. Es sei $M$ ein Wolf-Raum und $H$ eine kompakte, zusammenhängende Liegruppe, die isometrisch auf $M$ wirkt. Dann existiert mindestens einer der folgenden Orbittypen:

(i) ein komplexer Orbit,

(ii) ein totalgeodätischer quaternionaler Orbit,

(iii) eine $\mathbb{Z}_{2}$-Unterlagerung eines komplexen Orbits im Twistorraum von $M$. 


\section{Introduction}

The first systematic study of polar actions on Riemannian manifolds began with the paper A general theory of canonical forms of Palais and Terng [PaTe 1987]. Nevertheless a special case, namely the hyperpolar ones, were considered a long time before. For example Conlon has shown that hyperpolar actions (he called them actions admitting a $K$-transversal domain) are variationally complete [Co 1971], generalizing results of Bott and Samelson, who have proven that the adjoint action of a compact Lie group and the isotropy action of a compact symmetric space are variationally complete, these two actions being particularly simple examples of polar actions [BoSa 1958].

The case of polar representations has been analyzed by Dadok, who has classified them thereby proving that they are orbit equivalent to $s$-representations [Dad 1985]. Since the slice representation of an arbitrary polar action is again polar, this shows that the geometry of polar actions is intimately connected with the geometry of symmetric spaces. Actually most of the known examples of polar actions are given on symmetric spaces. Now all these examples on compact irreducible symmetric spaces of higher rank turned out to be even hyperpolar. The question whether this holds in full generality remains open, however the question has been answered for a large class of spaces. The first answer to this question was given on certain subclasses of Hermitian symmetric spaces. Podestà and Thorbergsson have classified polar actions on the complex quadric making use of their result that in this case the action is coisotropic, meaning that the sections are totally real submanifolds [PoTh 2002]. The same idea was used by Gori and Biliotti for the complex Grassmannian [BiGo 2005] and by Biliotti for the remaining Hermitian symmetric spaces of higher rank [Bi 2006]. It turned out that polar actions on irreducible higher rank Hermitian symmetric spaces of compact type are hyperpolar.

Recently Kollross finished the classification of polar actions on symmetric spaces of type I showing that polar actions are hyperpolar on these spaces if they are of higher rank. In principle his methods should also work for symmetric spaces of type II but the computational complexity becomes too large, so this case remains open.

The first part of this thesis is concerned with polar actions on Hermitian symmetric spaces. The main motivation for us was to supplement geometric ideas which might be helpful in a conceptual proof of the fact mentioned above making use of the rich geometrical structure of these spaces. In particular our results are independent of the classification results of polar actions on Hermitian symmetric spaces. Especially the existence of a momentum map for isometric 
actions turns out to be helpful. Recall that for a symmetric space the polarity of a compact Lie group action is equivalent to the integrability of the principal horizontal distribution [PaTe 1988]. On a Hermitian symmetric space the result of Podestà and Thorbergsson mentioned above implies that the principal horizontal distribution is totally real. We call a polar action on a Kähler manifold complex integrable if the complexified principal horizontal distribution is integrable. The main result of the first part is a reduction theorem. We reduce the acting group to a certain subtorus which is defined by the momentum map while reducing at the same time the codimension of the section. So let $M$ be an irreducible Hermitian symmetric space of compact type endowed with a complex integrable polar action of a compact connected Lie group $H$ and $\mu: M \rightarrow \mathfrak{h}^{*}$ a momentum map for the $H$-action. We will prove:

Theorem (Reduction Theorem). Let $p \in M$ be a regular point of the $H$-action, $\Sigma$ the section through $p$ and $T:=H_{\mu(p)} / H_{p}$. Then:

(i) $\Sigma^{\mathbb{C}}:=\overline{T^{\mathbb{C} p}}$ is a totally geodesic submanifold of $M$ which contains $\Sigma$ as a Lagrangian submanifold.

(ii) The T-action on $\Sigma^{\mathbb{C}}$ is polar with $\Sigma$ as a section.

(iii) The complexified section $\Sigma^{\mathbb{C}}$ splits as a product of complex projective spaces, i. e. $\Sigma^{\mathbb{C}}=\mathbb{P}_{n_{1}}(\mathbb{C}) \times \cdots \times \mathbb{P}_{n_{k}}(\mathbb{C})$.

In the next section we will give applications of this theorem. We obtain several corollaries. The first one is a criterion for the hyperpolarity of the $H$-action.

Corollary. The $H$-action is hyperpolar if and only if there exists a totally geodesic momentum fiber through a regular point of the $H$-action.

Moreover the Reduction Theorem enables us to show that the sections of the $H$-action are necessarily products of real projective spaces. Compare this also to Theorem 5.4 of [Kol 2006], where Kollross proves that a section of a polar action is covered by a product of spaces of constant curvature.

Corollary. Let $\Sigma$ be a section for the $H$-action on $M$. Then $\Sigma$ is isometric to a product of real projective spaces, i. e. $\Sigma=\mathbb{P}_{n_{1}}(\mathbb{R}) \times \cdots \times \mathbb{P}_{n_{k}}(\mathbb{R})$. The $H$-action is hyperpolar if and only if $n_{1}=\cdots=n_{k}=1$.

Observing that every polar action on a complex projective space is complex integrable this leads to a new conceptual proof of the fact that in this case a section must be a real projective space. This was first observed by Podestà and Thorbergsson in [PoTh 1999], where they classified polar actions on the rank one spaces.

Corollary. Let $X$ be a complex projective space and $H$ a connected compact Lie group acting polarly on $X$. Then the action is complex integrable and a section is isometric to a real projective space.

We also prove that complex integrable polar actions are provided by polar $\mathbb{C}$-asystatic ones. 
Proposition. Let $M$ be a compact homogeneous simply-connected irreducible Kähler manifold and $H \times M \rightarrow M$ a polar $\mathbb{C}$-asystatic action of a compact connected Lie group $H$. Then the $H$-action is complex integrable.

One of the main difficulties in a further analysis is to understand the geometry of the torus actions of $T=H_{\mu(p)} / H_{p}$ and $T^{\mathbb{C}}$. What is the relation between a regular point $p \in M$ and the torus $T=H_{\mu(p)} / H_{p}$, respectively $T^{\mathbb{C}}$ ? While the general case remains open due to fact that algebraic torus actions on generalized flag manifolds are only well understood if the torus in question is maximal, we obtain some further results for the series $C I$, i. e. $M=\operatorname{Sp}(n) / U(n)$. The key property of this spaces we will use is that they are symmetric spaces of maximal rank, i. e. rk $G / K=$ rk $G$. First of all we identify the generic torus orbit type for $M$ using results of Dabrowski [Da 1996].

Proposition. The generic torus orbit type for $M=G / K=\operatorname{Sp}(n) / \mathrm{U}(n)$, i. e. the generic torus orbit type of a maximal torus $T^{\mathbb{C}} \subset G^{\mathbb{C}}$ is isomorphic to the product of $n$ complex projective lines.

Note that this is the orbit type for the torus which is defined by the momentum map for the isotropy action on $M$, i. e. $T=K_{\mu(p)} / K_{p}$. It follows that for a hyperpolar action of maximal cohomogeneity the point $p$ is contained in the non-degenerate stratum $Z\left(T^{\mathbb{C}}\right)$ of the Torus $T^{\mathbb{C}}$. This is the stratum which is defined by the intersection of the big cells of all Borel subgroups in $G^{\mathbb{C}}$ which contain $T^{\mathbb{C}}$.

Proposition. Let $H \times M \rightarrow M$ be a complex integrable hyperpolar action with maximal cohomogeneity of a compact connected Lie group on $M=\operatorname{Sp}(n) / \mathrm{U}(n)$. Then every regular point $p \in M$ is contained in the non-degenerate stratum $Z\left(T^{\mathbb{C}}\right)$ of the complexification of the torus $T=H_{\mu(p)} / H_{p}$.

Maximal cohomogeneity means that $\operatorname{cohom}(H, M)=$ rk $G$. We conclude the chapter by applying these results and combinatorial properties of the momentum image to some low-dimensional examples.

Proposition. (i) Any complex integrable polar action of a connected compact Lie group on $M_{2}:=\mathrm{Sp}(2) / \mathrm{U}(2)$ is hyperpolar.

(ii) Any complex integrable polar action of a connected compact Lie group on $M_{3}:=\mathrm{Sp}(3) / \mathrm{U}(3)$ with maximal cohomogeneity, i. e. cohomogeneity equal to three is hyperpolar.

The last section of this thesis is concerned with polar actions on quaternionKähler symmetric spaces of compact type, i. e. the so-called Wolf spaces. The first result generalizes the main result of [PoTh 2002] to the quaternionic case.

Theorem. Let $M$ be a Wolf space and $H$ a connected compact Lie group acting polarly on $M$. Then the $H$-action is quaternion-coisotropic.

For this result we will make essential use of the classification result of Kollross [Kol 2006]. The main difficulty in generalizing the proof of the analogous result for the Kähler case is that we cannot guarantee the existence of a quaternionic orbit. However we will show: 
Theorem. Let $M$ be a Wolf space and $H$ a compact connected Lie group acting isometrically on $M$. Then the $H$-action admits at least one of the following orbits:

(i) a complex orbit,

(ii) a totally geodesic quaternionic orbit,

(iii) a $\mathbb{Z}_{2}$-quotient of a complex orbit in the twistor space $Z$ of $M$.

I would like to thank my teacher Professor Gudlaugur Thorbergsson, who supported me since the very beginning of my studies. Without his patience and constant encouragement this work would not have been possible.

Special thanks go to my parents and to my friend and colleague Dr. Oliver Goertsches. 


\section{Chapter 1}

\section{Polar actions}

\subsection{Introduction and examples}

Let $M$ be a complete Riemannian manifold and $G$ a compact connected Lie group acting isometrically on $M$. A submanifold $\Sigma \subset M$ is called a section if $\Sigma$ intersects every $G$-orbit and is orthogonal to the $G$-orbits in all common points. An isometric $G$-action that admits a section is called polar. A polar action, such that the section $\Sigma$ is flat, is called hyperpolar.

\section{Examples 1.1.1.}

- The standard representation of $\mathrm{SO}(n)$ on $\mathbb{R}^{n}$ is polar, a section simply being a line through the origin. The picture for $n=2$ explains the name polar.

- Let $G$ be a compact connected Lie group acting on itself by conjugation. Then the $G$-action is polar and any maximal torus of $G$ is a section.

- Let $M=G / K$ be a symmetric space. Then the natural action of $K$ on $M$ and the isotropy representation of $K$ on $T_{e K} M$ are polar. In the first case a section is given by a maximal flat totally geodesic submanifold through the point $e K$, while in the second case the section is a maximal abelian subspace of $T_{e K} M$.

- Let $M=G / K$ be a symmetric space and $H \subset G$ a symmetric subgroup. Then the action of $H$ on $M$ is hyperpolar. These examples are called Hermann actions.

Actually all these examples are hyperpolar ones. So let us give one polar but not hyperpolar example, which will be important in the following.

Example 1.1.2. Let $M=\mathrm{SU}(n+1) / \mathrm{S}(\mathrm{U}(1) \times \mathrm{U}(n))$ be the complex projective space $\mathbb{P}_{n}(\mathbb{C})$ and $T \subset \mathrm{SU}(n+1)$ a maximal torus. Then the $T$-action on $M$ is polar, but not hyperpolar. A section is isometric to $\mathbb{P}_{n}(\mathbb{R})$. 


\subsection{Basic properties}

In the following we will collect some well-known facts about polar actions. The main references are [PaTe 1987] and [PaTe 1988]. We will only prove that sections are automatically totally geodesic since there is no satisfactory proof in the literature.

First of all it is easy to see that for all $g \in G$ the translated section $g \Sigma$ is again a section. Together with the fact that $\Sigma$ intersects every orbit, we see that there exists a section through every point of $M$. If $p \in M$ is a regular point of the $G$-action then the section through $p$ is uniquely determined. It is just the image of the normal space of the orbit $G \cdot p$ under the exponential map of $M$. Given any polar action, the slice representation, i. e. the restriction of the isotropy representation to the normal space, is again polar. If $\Sigma$ is a section through $p$ then $T_{p} \Sigma$ is a section for the slice representation. The orbit structure of polar representations is well understood. Dadok [Dad 1985] has proven that every polar representation of a compact connected Lie group is orbit equivalent to the isotropy representation of a symmetric space. Together with the facts about the slice representation mentioned above this turns out to be an important tool for the analysis of polar actions.

Proposition 1.2.1. Let $(M, g)$ be a complete Riemannian manifold and $G$ a compact Lie group acting polarly on $M$. Then the sections for the $G$-action are totally geodesic.

Before we come to the proof we need the following two lemmas:

Lemma 1.2.2. Let $G \times M \rightarrow M$ be a polar action and $\Sigma$ a section. Then the dimension of $\Sigma$ equals the cohomogeneity of the $G$-action.

Proof. First we note that it is enough to show that $\operatorname{dim} \Sigma \geq \operatorname{codim}(G \cdot q)$ for a regular point $q \in M$. Since $\Sigma$ intersects every orbit of $G$, the map

$$
\phi: G \times \Sigma \rightarrow M, \quad(g, p) \mapsto g p
$$

is onto. Let $n+k=\operatorname{dim} M$, where $k$ is the cohomogeneity of the $G$-action. The Theorem of Sard implies that the maximal rank of $\phi$ equals $n+k$ : Suppose $\operatorname{rank}\left(d \phi_{x}\right)<n+k$ for all $x \in G \times \Sigma$, then the set $\phi(G \times \Sigma)$ has measure zero in $M$, but this is a contradiction since $\phi(G \times \Sigma)=M$.

Next we choose a point $q \in M$ which is regular for the $G$-action and which is also a regular value of $\phi$. This choice is clearly possible, since $M_{r}$ is open and dense in $M$ and the set of non regular values of $\phi$ has measure zero.

Let $(g, p) \in \phi^{-1}(q)$, then $d \phi_{(g, p)}: T_{(g, p)}(G \times \Sigma) \rightarrow T_{q} M$ is onto and we have the decompositions

$$
T_{(g, p)}(G \times \Sigma)=T_{g} G \times T_{p} \Sigma=T_{g} G \times\{0\} \oplus\{0\} \times T_{q} \Sigma
$$

and

$$
T_{q} M=T_{q} G \cdot q \oplus N_{q},
$$

where $N_{q}$ denotes the normal space of the orbit $G \cdot q$. 
We claim that $d \phi_{(g, p)}\left(T_{g} G \times\{0\}\right) \subset T_{q} G \cdot q$ : If $(v, 0) \in T_{q} G \times\{0\}$ and $\gamma=\left(\gamma_{1}, \gamma_{2}\right):(-\epsilon, \epsilon) \rightarrow G \times \Sigma$ is a path with $\gamma(0)=(g, p), \dot{\gamma}_{1}(0)=v, \gamma_{2}(t)=p$ for all $t \in(-\epsilon, \epsilon)$, then (note that $G \cdot p=G \cdot q$ )

$$
d \phi_{(g, p)}(v)=\left.\frac{d}{d t}\right|_{t=0}(\phi \circ \gamma)(t)=\left.\frac{d}{d t}\right|_{t=0} \gamma_{1}(t) \cdot \gamma_{2}(t)=\left.\frac{d}{d t}\right|_{t=0} \gamma_{1}(t) \cdot p \in T_{q} G \cdot q
$$

Since $d \phi_{(g, p)}$ is onto, we must have $\operatorname{dim} d \phi_{(g, p)}\left(\{0\} \times T_{p} \Sigma\right) \geq N_{q}$ and hence $\operatorname{dim} \Sigma \geq \operatorname{dim} N_{q}=\operatorname{codim}(G \cdot q)$.

Lemma 1.2.3. Let $\Sigma$ be a section for the $G$-action on $M$. Then the set $\Sigma_{r}:=\Sigma \cap M_{r}$ is open and dense in $\Sigma$. Here $M_{r}$ denotes the set of regular points of the G-action.

Proof. Let $d$ be the dimension of a principal orbit. Then the union of all nonprincipal orbits of dimension at most $t$ is a closed set of dimension at most $n-d+t-1$. This is a result of Montgomery, Samelson, Yang and Zippin (cf. [MSZ 1956],[MSY 1956]). First of all it is clear that $\Sigma_{r}$ is open in $\Sigma$ since $\Sigma$ carries the relative topology.

Now suppose that $\Sigma_{r}$ is not dense. This means that there exists an open set $U \subset \Sigma$, which contains only non regular points. We set $l:=\operatorname{dim} \Sigma, n:=\operatorname{dim} M$ and choose $k \geq 0$ such that $n-l-k$ is the maximal dimension of the orbits that meet $U$. By shrinking $U$ we can assume that every orbit that meets $U$ has dimension $n-l-k$. Let $B$ denote the union of all non-principal orbits of $M$ with dimension at most $n-l-k$. We can write $B=\bigcup_{i \in I} M_{i}$, where each $M_{i}$ is a connected component of some orbit type in $M$, so $B$ is a stratified space and each $M_{i}$ is stratum. It follows that

$$
\operatorname{dim} M_{i} \leq n-(n-l)+(n-l-k)-1=n-k-1
$$

for each $i \in I$.

We clearly have $U \subset B$ and we claim that there exists an open subset $V \subset U$ such that $V$ is completely contained in some stratum $M_{i_{0}}$. The sets $U \cap \bar{M}_{i}$ are closed in $U$ and we have $U=\bigcup_{i \in I} U \cap \bar{M}_{i}$, thus the Theorem of Baire implies that there exists an open set $\tilde{V} \subset U$ with $\tilde{V} \subset \bar{M}_{k}$ for some $k \in I$. If $\tilde{V}$ is not completely contained in the boundary of $\bar{M}_{k}$, then we can shrink $\tilde{V}$ to find a set $V$ as claimed. So let us suppose that $\tilde{V}$ is completely contained in $\partial \bar{M}_{k}$. The definition of a stratification now implies that $\tilde{V} \subset \bigcup_{j \in J} M_{j}, J \subset I$ and $\operatorname{dim} M_{j}<\operatorname{dim} M_{k}$ for each $j \in J$. Note that the dimension of $U$ is a lower bound for $\operatorname{dim} \overline{M_{j}}$ if $U \subset \overline{M_{j}}$. This means that if we iterate this procedure, it terminates after finitely many steps with an open set $\tilde{V}$ and a stratum $M_{i_{0}}$ such that $\tilde{V} \subset \bar{M}_{i_{0}}$ and $\operatorname{dim} \tilde{V}=\operatorname{dim} \bar{M}_{i_{0}}$. Now it is clear that $\tilde{V}$ cannot be completely contained in the boundary of $\bar{M}_{i_{0}}$ and we can shrink $\tilde{V}$ to an open set $V$ as claimed.

It follows $T_{p} \Sigma \subset T_{p} M_{i_{0}}$ for any point $p \in V$. If we write $T_{p} M_{i_{0}}=T_{p} G \cdot p \oplus N_{p}$, where $N_{p}$ is the normal space of $T_{p} G \cdot p$ in $T_{p} M_{i_{0}}$, then it even follows $T_{p} \Sigma \subset N_{p}$. But this is a contradiction to the fact that

$$
\operatorname{dim} N_{p}=\operatorname{dim} T_{p} M_{i_{0}}-\operatorname{dim} T_{p} G \cdot p \leq(n-k-1)-(n-l-k)=l-1 .
$$


Remark 1.2.4. Note that this lemma is much easier to prove if one is allowed to use that $\Sigma$ is totally geodesic.

Proof of Proposition 1.2.1. Since the $G$-action is polar, the principal horizontal distribution, i. e. the horizontal distribution of the Riemannian submersion

$$
\pi: M_{r} \rightarrow M_{r} / G
$$

is integrable and a leaf is given by a connected component of $\Sigma_{r}$. It is well known that the leaves of an integrable horizontal distribution of a Riemannian submersion are totally geodesic, so $\Sigma_{r}$ is totally geodesic. Since $\Sigma_{r}$ is dense in $\Sigma$ the same holds for $\Sigma$. 


\section{Chapter 2}

\section{Polar actions on Hermitian symmetric spaces}

\section{$2.1 \quad$ Introduction}

\subsubsection{Hermitian symmetric spaces as generalized flag manifolds}

The main references for this section are [He 1962], [Bo 1991], [Hu 1975]. Let $M$ be an irreducible Hermitian symmetric space of compact type. Then $M$ can be realized as a quotient $G / K$, where $G$ is a connected compact simple Lie group with trivial center and $K$ is a maximal connected proper subgroup of $G$ with discrete center. There are two other important geometric realizations of $M$, which we will use in the sequel: one as an adjoint orbit and the other as a generalized flag manifold.

Let $\mathfrak{g}=\mathfrak{k} \oplus \mathfrak{m}$ be the corresponding Cartan decomposition of $\mathfrak{g}$. Then there exists an element $z$ in the center of $\mathfrak{k}$ such that $\operatorname{ad}_{z}: \mathfrak{m} \rightarrow \mathfrak{m}$ gives the complex structure of $M$. Since $z$ lies in the center of $\mathfrak{k}$, it is easy to see that $K$ is the stabilizer in $z$ for the adjoint action of $G$ on $\mathfrak{g}$ and hence $M=\operatorname{Ad}(G) \cdot z$. This description as an adjoint orbit will guarantee that later on all isometric actions of Lie subgroups of $G$ on $M$ are automatically Hamiltonian.

On the other hand let $G^{\mathbb{C}}$ denote the complexification of $G$. Then $M$ is a $G^{\mathbb{C}}$-homogeneous space and hence $M=G^{\mathbb{C}} / P$, where $P$ is a parabolic subgroup of $G^{\mathbb{C}}$.

There exists the following root-theoretical description of $P$ : We fix a Cartan subalgebra $\mathfrak{t}^{\mathbb{C}} \subset \mathfrak{g}^{\mathbb{C}}$ and consider the root system $\Delta=\Delta\left(\mathfrak{g}^{\mathbb{C}}, \mathfrak{t}^{\mathbb{C}}\right)$ of $\mathfrak{g}^{\mathbb{C}}$ with respect to $\mathfrak{t}^{\mathbb{C}}$. Let $\Delta^{+}$denote the set of positive roots for some ordering of $\Delta$ and $\pi \subset \Delta^{+}$the set of simple roots. Then

$$
\mathfrak{b}=\mathfrak{t}^{\mathbb{C}} \oplus \bigoplus_{\alpha \in \Delta^{+}} \mathfrak{g}_{\alpha}^{\mathbb{C}}
$$

is a Borel subalgebra and the parabolic algebras $\mathfrak{p}$ with $\mathfrak{p} \supset \mathfrak{b}$ are in one-to-one correspondence with subsets $\pi_{P}$ of $\pi$. The parabolic subgroups corresponding to Hermitian symmetric spaces are maximal ones and hence in this case the subset $\pi_{P}$ arises from $\pi$ by deleting one root. More precisely: The sets 
$\pi_{P}=\pi \backslash\{\alpha\}$ which give rise to Hermitian symmetric spaces are characterized by the condition that the coefficient of $\alpha$ in the sum description of the highest root of $\Delta$ is one. Let $\omega_{i}$ denote the fundamental dominant weight corresponding to the root $\alpha_{i} \in \pi$ and $V$ a $G^{\mathbb{C}}$-module with heighest weight

$$
\delta=\sum_{\alpha_{i} \in \pi_{P}} \omega_{i} \text { and heighest vector } v_{\delta} \in V
$$

then we have

$$
G^{\mathbb{C}} / P \cong G^{\mathbb{C}} \cdot\left[v_{\delta}\right] \subset \mathbb{P}(V) .
$$

Let $B \subset G^{\mathbb{C}}$ be a Borel subgroup. The $B$-orbits in $M$ are called Schubert cycles. From the Bruhat-decomposition of $G^{\mathbb{C}}$ it follows that there exists a unique open and dense $B$-orbit in $M$, which is called the big cell of $B$ in $M$. The Weyl group $W:=N_{G}^{\mathbb{C}}\left(T^{\mathbb{C}}\right) / T^{\mathbb{C}}$ operates simply transitive on the set $\mathcal{B}^{T^{\mathbb{C}}}$ of all Borel subgroups containing $T^{\mathbb{C}}$, so we can write

$$
\mathcal{B}^{T^{\mathbb{C}}}=\left\{w B^{-} w^{-1} \mid w \in W\right\},
$$

where $B^{-}$is the opposite Borel subgroup of $B$, i. e. $B \cap B^{-}=T^{\mathbb{C}}$. For each of this Borel subgroups one gets one big cell and the intersection of all these big cells is called the non-degenerate stratum $Z\left(T^{\mathbb{C}}\right)$. The big cell for $B^{-}$is just the $B^{-}$-orbit through the origin $e P \in G / P$ and hence one has the following description:

$$
Z\left(T^{\mathbb{C}}\right)=\bigcap_{w \in W} w B^{-} w^{-1} w P=\bigcap_{w \in W} w B^{-} P=\bigcap_{w \in W / W_{P}} w B^{-} P
$$

where $W_{P}$ is the subgroup of $W$ generated by reflections $\alpha \in \pi_{P}$ or alternatively $W_{P}=N_{P}\left(T^{\mathbb{C}}\right) / T^{\mathbb{C}}$.

\subsubsection{Torus actions and toric varieties}

The main reference for this section is [Oda 1988]. Since this is non-standard material from the differential geometric viewpoint, we allow ourselves to be a little more elaborate. The key idea is that toric varieties are in one-to-one correspondence to combinatorial objects called fans, which are families of certain cones. Let us first define the notion of a toric variety:

Definition 2.1.1. Let $X$ be an irreducible normal scheme $X$ over $\mathbb{C}$ which is locally of finite type over $\mathbb{C}$ and seperated. Then $X$ is called a toric variety, if it containes an algebraic torus $T^{\mathbb{C}}$ as an open set, such that $X$ admits an algebraic action $T^{\mathbb{C}} \times X \rightarrow X$ which extends the group multiplication $T^{\mathbb{C}} \times T^{\mathbb{C}} \rightarrow T^{\mathbb{C}}$.

Remark 2.1.2. It is clear that any irreducible complex manifold admitting an almost homogeneous action of an algebraic torus is a toric variety.

We now would like to define the involved combinatorial objects. Let $N$ be a free $\mathbb{Z}$-module of rank $r, M$ its dual module, i. e. $M:=\operatorname{Hom}_{\mathbb{Z}}(N, \mathbb{Z})$ and 
$\langle\cdot, \cdot\rangle: M \times N \rightarrow \mathbb{Z}$ the canonical pairing. We define an algebraic torus $T_{N}$ by setting

$$
T_{N}:=N \otimes_{\mathbb{Z}} \mathbb{C}^{*}=\operatorname{Hom}_{\mathbb{Z}}\left(M, \mathbb{C}^{*}\right),
$$

then $T_{N}$ is isomorphic to $\mathbb{C}^{*}$ and $N$ can be considered as its group of oneparameter subgroups, while $M$ is its group of characters. An element $n \in N$ gives rise to a one-parameter subgroup $\gamma_{n}: \mathbb{C}^{*} \rightarrow T_{N}$ by setting

$$
\gamma_{n}(\lambda)(m):=\lambda^{\langle m, n\rangle} \text { for all } \lambda \in \mathbb{C}^{*} \text { and } m \in M
$$

and an element $m \in M$ defines a charakter $\chi(m): T_{N} \rightarrow \mathbb{C}^{*}$ by

$$
\chi(m)(t):=t(m) \text { for all } t \in T_{N} .
$$

Let $N_{\mathbb{R}}$ and $M_{\mathbb{R}}$ be the mutually dual $\mathbb{R}$-vector spaces

$$
N_{\mathbb{R}}:=N \otimes_{\mathbb{Z}} \mathbb{R} \text { and } M_{\mathbb{R}}:=M \otimes_{\mathbb{Z}} \mathbb{R}=\operatorname{Hom}_{\mathbb{Z}}(N, \mathbb{R}) .
$$

Definition 2.1.3. A convex polyhedral cone $\sigma$ is a subset $\sigma \subset N_{\mathbb{R}}$ such that there exist elements $n_{1}, \ldots, n_{s} \in N_{\mathbb{R}}$ with

$$
\sigma=\mathbb{R}_{\geq 0} n_{1}+\cdots+\mathbb{R}_{\geq 0} n_{s} .
$$

The cone $\sigma$ is called rational, if $n_{1}, \ldots, n_{s}$ can be chosen to lie in $N$. The dual cone $\sigma^{\vee} \subset M_{\mathbb{R}}$ is defined by

$$
\sigma^{\vee}:=\left\{m \in M_{\mathbb{R}} \mid\langle m, n\rangle \geq 0, \text { for all } n \in \sigma\right\} .
$$

A subset $\tau \subset \sigma$ is called a face of $\sigma$, if there exists an element $m_{0} \in M_{\mathbb{R}}$ such that $m_{0} \in \sigma^{\vee}$ and

$$
\tau=\sigma \cap\left\{m_{0}\right\}^{\perp}:=\left\{n \in \sigma \mid\left\langle m_{0}, n\right\rangle=0\right\} .
$$

A fan $\Delta$ is a family of convex polyhedral cones in $N_{\mathbb{R}}$ subject to following conditions:

(i) Each $\sigma \in \Delta$ is rational.

(ii) Each $\sigma \in \Delta$ is strongly convex, meaning that $\sigma \cap(-\sigma)=\{0\}$.

(iii) Every face of $\sigma$ belongs to $\Delta$.

(iv) For $\sigma, \sigma^{\prime} \in \Delta$, the intersection $\sigma \cap \sigma^{\prime}$ is a face of $\sigma$ and $\sigma^{\prime}$.

We now come to the fundamental result in this theory:

Theorem 2.1.4. Via a covariant functor $(N, \Delta) \rightarrow T_{N} \mathrm{emb}(\Delta)$ the category of fans is equivalent to the category of toric varieties over $\mathbb{C}$.

Remark 2.1.5. Here $T_{N} \mathrm{emb}(\Delta)$ is defined as the union of certain sets $U_{\sigma}$ :

$$
T_{N} \operatorname{emb}(\Delta):=\bigcup_{\sigma \in \Delta} U_{\sigma}, \text { where }
$$

$U_{\sigma}:=\left\{u: M \cap \sigma^{\vee} \rightarrow \mathbb{C} \mid u(0)=1, u\left(m+m^{\prime}\right)=u(m) u\left(m^{\prime}\right)\right.$ for $\left.m, m^{\prime} \in M \cap \sigma^{\vee}\right\}$.

One can prove that these sets can be identified with algebraic subsets of $\mathbb{C}^{n}$ and the conditions (i)-(iv) in the definition of a fan guarantee that these sets glue together to define a toric variety. 
Example 2.1.6. (i) Let $N=\mathbb{Z}$ and $\Delta:=\{\sigma,-\sigma,\{0\}\}$ with $\sigma:=\mathbb{R}_{\geq 0} \subset N_{\mathbb{R}}$. It is clear that $\Delta$ is a fan. One easily shows that $U_{\sigma} \cong \mathbb{C} \cong U_{-\sigma}$ and $U_{\{0\}}=\mathbb{C}^{*}$. Hence one obtains $T_{N} \mathrm{emb}(\Delta)=\mathbb{P}_{1}(\mathbb{C})$ by gluing two copies of $\mathbb{C}$ along their common open set $\mathbb{C}^{*}$.

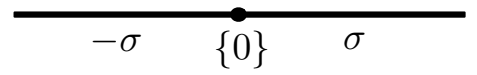

Figure 2.1: Fan of $\mathbb{P}_{1}(\mathbb{C})$

(ii) For two fans $\Delta, \Delta^{\prime}$ in $N$ and $N^{\prime}$ one has

$$
T_{N \times N^{\prime}} \operatorname{emb}\left(\Delta \times \Delta^{\prime}\right) \cong T_{N} \operatorname{emb}(\Delta) \times T_{N^{\prime}} \operatorname{emb}\left(\Delta^{\prime}\right)
$$

and hence Figure 2.2 describes the fan of $\mathbb{P}_{1}(\mathbb{C}) \times \mathbb{P}_{1}(\mathbb{C})$.

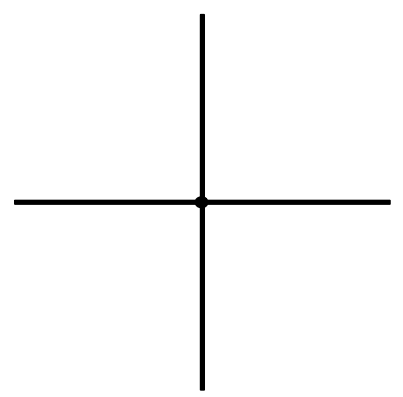

Figure 2.2: Fan of $\mathbb{P}_{1}(\mathbb{C}) \times \mathbb{P}_{1}(\mathbb{C})$

\subsubsection{The symplectic geometry of Hermitian symmetric spaces}

Let $(M, \omega)$ be a symplectic manifold and $H$ a compact connected Lie group acting on $M$ by symplectomorphisms, i. e. $g^{*} \omega=\omega$ for all $g \in H$. The action is called Hamiltonian if there exists a momentum map $\mu: M \rightarrow \mathfrak{h}^{*}$, which is by definition an $H$-equivariant map such that

$$
\iota_{X_{\xi}} \omega=d \mu^{\xi} \text { for all } \xi \in \mathfrak{h},
$$

where $\mu^{\xi}: M \rightarrow \mathbb{R}$ is defined by $\mu^{\xi}(x):=\mu(x)(\xi)$. Here $X_{\xi}$ denotes the vector field on $M$ induced by $\xi \in \mathfrak{h}$ and $\iota_{X_{\xi}} \omega$ the contraction of $\omega$ by $X_{\xi}$. Let $x \in M$ and $d \mu_{x}: T_{x} M \rightarrow T_{\mu(x)} \mathfrak{h}^{*}$ be the differential of $\mu$. We then have

$$
\text { ker } d \mu_{x}=\left(T_{x} H \cdot x\right)^{\perp_{\omega}}:=\left\{v \in T_{x} M \mid \omega(v, w)=0 \text { for all } w \in T_{x} H \cdot x\right\} .
$$

In general the existence of a momentum map is not clear, however there are some special situations, where the existence of $\mu$ can be guaranteed.

Let $M$ be a coadjoint orbit for a Lie group $G$. Then the momentum map for $G$ is just the inclusion of $M$ into $\mathfrak{g}^{*}$. Any action of a Lie subgroup $H \subset G$ is again Hamiltonian and the momentum map for the $H$-action is just the projection on $\mathfrak{h}^{*}$. Another important situation is the following: Let $H$ be a compact Lie group acting in a Hamiltonian way on $M$ and $N \subset M$ a submanifold which 
is invariant under $H$. Then the $H$-action on $M$ is again Hamiltonian and the corresponding momentum map is just the restriction of the original momentum map to $N$.

Let $N \subset M$ be a submanifold. We call $N$ a coisotropic submanifold if

$$
T_{p} N^{\perp_{\omega}} \subset T_{p} N
$$

for all points $p \in N$. Let $J \in \operatorname{End}(T M)$ be a compatible almost complex structure, i. e. $\omega(J X, J Y)=\omega(X, Y)$ and $g(X, Y):=\omega(X, J Y)$ defines a Riemannian metric on $M$. Then condition (2.1) can be equivalently formulated as

$$
\nu_{p} N \perp_{g} J \nu_{p} N
$$

for all $p \in N$, where $\nu_{p} N$ denotes the normal space of $N$ in $M$, i. e. the normal bundle of $N$ is totally real. We call a Lie group action on $M$ coisotropic, if all principal orbits are coisotropic.

Let $M$ be now a Hermitian symmetric space of compact type. Then any isometric action on $M$ is Hamiltonian, since $M$ can be realized as an adjoint orbit. The following theorem links the theory of polar actions to that of coisotropic ones.

Theorem 2.1.7 (PoTh 2002). A polar action on an irreducible compact homogeneous simply-connected Kähler manifold is coisotropic.

So in other words the sections are totally real submanifolds of $M$.

Another important theorem that characterizes coisotropic actions on compact Kähler manifolds is the following Equivalence Theorem of Huckleberry and Wurzbacher [HuWu 1990].

Theorem 2.1.8 (HuWu 1990). Let $M$ be a connected compact Kähler manifold with an isometric and Hamiltonian action of a connected compact group $H$. Then the following conditions are equivalent:

(i) The H-action is coisotropic.

(ii) The momentum map $\mu: M \rightarrow \mathfrak{h}^{*}$ separates orbits.

(iii) The cohomogoneity of the $H$-action is equal to the difference between the rank of $H$ and the rank of a regular isotropy subgroup of $H$.

(iv) The space $C^{\infty}(M)^{H}$ of $H$-invariant functions on $M$ is abelian with respect to the Poisson bracket.

(v) The Kähler manifold $M$ is projective algebraic, $H^{\mathbb{C}}$-almost homogeneous and a spherical embedding of the open $H^{\mathbb{C}}$-orbit.

Remark 2.1.9. The points (ii) and (iii) will be of special importance for our work. 
Now the combined use of these two theorems allows one to show that polar actions are hyperpolar on higher rank Hermitian symmetric spaces: Use (iii) of the Equivalence Theorem to classify the coisotropic actions and then determine under these actions the polar ones. This has been carried out for the first time in [PoTh 1999] for the complex quadric. Then Biliotti and Gori did this for the complex Grassmannians [BiGo 2005] and finally Biliotti [Bi 2006] solved the remaining cases. Of course here we have to mention the recent result of Kollross [Kol 2006] who completed the classification of polar actions on compact irreducible symmetric spaces of type I, thereby solving the general case. We would like to point out that the results in the following are completely independent from these classification results. Actually the main motivation for this work was to supplement geometric ideas which might be helpful in a conceptual proof of this fact. 


\subsection{The Reduction Theorem}

For a polar action on a Riemannian manifold the normal distribution to the principal orbits is integrable, a leaf is given by the intersection of a section with the set of regular points of the action. Now on a Kähler manifold the main result of [PoTh 2002] shows that the sections are totally real submanifolds, so it makes sense to define the following notion:

Definition 2.2.1. Let $M$ be an irreducible compact homogeneous simply-connected Kähler manifold and $H$ a compact connected Lie group acting polarly on $M$. We call the $H$-action complex integrable if and only if the distribution

$$
\mathcal{D}_{q}:=T_{q} \Sigma_{q} \oplus J T_{q} \Sigma_{q}, \quad q \in M_{r}
$$

is integrable. Here $M_{r}$ denotes the set of regular points of the $H$-action and $\Sigma_{q}$ is the unique section through $q$.

The first observation concerning a complex integrable polar action is the following:

Lemma 2.2.2. Let $H \times M \rightarrow M$ be a complex integrable polar action. Then the leaves of the distribution $\mathcal{D}$ are automatically totally geodesic.

Proof. Let $X, Y$ be two local sections of $\mathcal{D}$. We have to prove that the normal part of $\nabla_{X} Y$ with respect to $\mathcal{D}$ vanishes. Here $\nabla$ denotes the Levi-Civita connection of $M$. We write $X=X_{1}+J X_{2}$ and $Y=Y_{1}+J Y_{2}$ with $X_{i}, Y_{i}$ sections of $\mathcal{D}$ sucht that $X_{i}(q), Y_{i}(q) \in T_{q} \Sigma_{q}$. We then get

$$
\begin{aligned}
\nabla_{X} Y & =\nabla_{X_{1}} Y_{1}+\nabla_{X_{1}} J Y_{2}+\nabla_{J X_{2}} Y_{1}+\nabla_{J X_{2}} J Y_{2} \\
& =\nabla_{X_{1}} Y_{1}+J \nabla_{X_{1}} Y_{2}+\left[J X_{2}, Y_{1}\right]+J \nabla_{Y_{1}} X_{2}+J\left(\left[J X_{2}, Y_{2}\right]+J \nabla_{Y_{2}} X_{2}\right)
\end{aligned}
$$

which shows that $\nabla_{X} Y$ is tangent to $\mathcal{D}$ since the sections are totally geodesic and the distribution $\mathcal{D}$ is assumed to be integrable.

Examples of complex integrable polar actions are provided by polar actions which are complex asystatic. Recall that an isometric Lie group action is called asystatic if the isotropy representation of a principal isotropy subgroup has no non-trivial fixed vectors on the tangent space of the corresponding orbit. In [AlAl 1993] it is proved that asystatic actions are polar and a section is given by the fixed point set of a principal isotropy subgroup. Now on a Kähler manifold there is the related concept of a $\mathbb{C}$-asystatic action. This concept is introduced first in [PoTh 2002] and systematically studied in [GoPo 2006], where such actions are called symplectically asystatic, but for polar actions we prefer the notion $\mathbb{C}$-asystatic as we will explain. 
The following result is proved in [GoPo 2006]:

Proposition 2.2.3. Let $M$ be Kähler manifold endowed with an effective Hamiltonian action of a compact group $K$. Let $(L)$ be a principal isotropy type, $\bar{M}$ a core and $c$ the cohomogeneity of the $K$-action. Then

(i) $\operatorname{dim} \bar{M} \geq c+\operatorname{rk} K-\operatorname{rk} L$;

(ii) the equality in (i) holds if and only if the group $\left(N_{K}(L) / L\right)^{\circ}$ is abelian.

The core of the $K$-action is defined as the closure of $M^{L} \cap M_{r}$, where $M^{L}$ denotes the fixed point set of $L$. Now Gori and Podestà call the $K$-action symplectically asystatic if in (i) equality holds.

Let $H$ be now a compact connected Lie group acting polarly on an irreducible compact homogeneous simply-connected Kähler manifold. Note that for this action, which is coisotropic, $(i)$ reduces to

$$
\operatorname{dim} \bar{M} \geq 2 c .
$$

Let $p \in M$ be a regular point and $\Sigma$ the section through $p$. Then the tangent space of the $H$-orbit through $p$ splits as

$$
T_{p} H \cdot p=J T_{p} \Sigma \oplus\left(T_{p} H \cdot p \cap J T_{p} H \cdot p\right) .
$$

Assume now that in (i) equality holds. Then this means that the isotropy $H_{p}$ has no non-trivial fixed vector on the maximal holomorphic subspace $T_{p} H \cdot p \cap J T_{p} H \cdot p$ of $T_{p} H \cdot p$, so we define:

Definition 2.2.4. The $H$-action is called $\mathbb{C}$-asystatic if a principal isotropy subgroup has no non-trivial fixed vector on the maximal holomorphic subspace of the tangent space of the corresponding orbit.

The following is an example of a polar $\mathbb{C}$-asystatic action (cf. [BiGo 2005]): Let $M=G / K$ be the complex quadric $Q_{n}=\mathrm{SO}(n+2) / \mathrm{SO}(2) \times \mathrm{SO}(n)$. Then the $K$-action on $M$ is $\mathbb{C}$-asystatic. A principal isotropy subgroup $L$ is given by $\mathbb{Z}_{2} \times \mathrm{SO}(n-2)$ and $\left(N_{K}(L) / L\right)^{\circ}=\mathrm{SO}(2)^{2}$ is abelian.

We are interested in $\mathbb{C}$-asystatic actions because of the following proposition:

Proposition 2.2.5. A polar $\mathbb{C}$-asystatic action is complex integrable and a leaf $S_{p}$ through a point $p \in M_{r}$ is given by the intersection of $M_{r}$ with the connected component of the fixed point set of $H_{p}$ which contains $p$.

Proof. Let $S_{p}$ denote the intersection of $M_{r}$ with the connected component of the fixed point set of $H_{p}$ which contains $p$. Since $p$ is regular, $H_{p}$ acts trivially on $T_{p} \Sigma_{p} \oplus J T_{p} \Sigma$ and hence $T_{p} S_{p} \supset T_{p} \Sigma_{p} \oplus J T_{p} \Sigma_{p}$. On the other hand $T_{p} M$ splits as

$$
T_{p} M=T_{p} H \cdot p \oplus T_{p} \Sigma_{p}=J T_{p} \Sigma_{p} \oplus\left(T_{p} H \cdot p \cap J T_{p} H \cdot p\right) \oplus T_{p} \Sigma_{p}
$$

and we have seen that $H_{p}$ has no fixed points on $T_{p} H \cdot p \cap J T_{p} H \cdot p$, so we actually have $T_{p} S_{p}=T_{p} \Sigma_{p} \oplus J T_{p} \Sigma_{p}$. 
The next step is to show that we have $T_{x} S_{p}=T_{x} \Sigma_{x} \oplus J T_{x} \Sigma_{x}$ for an arbitrary point $x \in S_{p}$. Since $x$ is a regular point in the fixed point set of $H_{p}$, we have $H_{x}=H_{p}$. Using the same notations and arguments as above we have $T_{x} S_{x}=T_{x} \Sigma_{x} \oplus J T_{x} \Sigma_{x}$, but $M^{H_{x}}=M^{H_{p}}$ and hence $S_{x}=S_{p}$ and the claim follows.

From now on we assume $M=G / K$ to be an irreducible Hermitian symmetric space of compact type and $H$ a connected compact Lie group. Moreover the $H$-action on $M$ is assumed to be polar and complex integrable. Let $\mu: M \rightarrow \mathfrak{h}$ be a momentum map for the $H$-action, which clearly exists, since $M$ can be realized as the coadjoint orbit of the full isometry group of $M$. Since $H$ is compact, we have identified $\mathfrak{h}$ with its dual. In this section we will prove the following Reduction Theorem for complex integrable polar actions on Hermitian symmetric spaces:

Theorem 2.2.6 (Reduction Theorem). Let $p \in M$ be a regular point for a complex integrable polar $H$-action, $\Sigma$ the section through $p$ and $T:=H_{\mu(p)} / H_{p}$. Then:

(i) $\Sigma^{\mathbb{C}}:=\overline{T^{\mathbb{C}} p}$ is a totally geodesic submanifold of $M$ which contains $\Sigma$ as a Lagrangian submanifold.

(ii) The T-action on $\Sigma^{\mathbb{C}}$ is polar with $\Sigma$ as a section.

(iii) The complexified section $\Sigma^{\mathbb{C}}$ splits as a product of complex projective spaces, i. e. $\Sigma^{\mathbb{C}}=\mathbb{P}_{n_{1}}(\mathbb{C}) \times \cdots \times \mathbb{P}_{n_{k}}(\mathbb{C})$.

Remark 2.2.7. The notation $T=H_{\mu(p)} / H_{p}$ is somewhat a short hand notation. We will give a precise definition after Lemma 2.2.12. This theorem can be viewed as a reduction theorem in the following sense, since it allows two simplifications. First of all it reduces the codimension of $\Sigma$ to the minimal possible if one wants the ambient space to stay complex. Next it reduces the acting group in a significant way: Starting with a polar action of a general Lie group we arrive at a torus.

As applications of the Reduction Theorem we will prove several corollaries. The first one is a criterion for the hyperpolarity of the $H$-action in terms of the fiber of the momentum map:

Corollary 2.2.8. The $H$-action is hyperpolar if and only if there exists a totally geodesic momentum fiber through a regular point of the $H$-action.

The next corollary gives a description of $\Sigma$ itself:

Corollary 2.2.9. Let $\Sigma$ be a section for the $H$-action on $M$. Then $\Sigma$ is isometric to a product of real projective spaces, i. e. $\Sigma=\mathbb{P}_{n_{1}}(\mathbb{R}) \times \cdots \times \mathbb{P}_{n_{k}}(\mathbb{R})$. The $H$-action is hyperpolar if and only if $n_{1}=\cdots=n_{k}=1$.

This yields observing that on a complex projective space every polar action is complex integrable a new conceptual proof of the fact that in this case the sections are necessarily isometric to real projective spaces. This was first observed in [PoTh 1999]. 
Corollary 2.2.10. Let $X$ be a complex projective space and $H$ a connected compact Lie group acting polarly on $X$. Then the action is complex integrable and a section is isometric to a real projective space.

The proofs of these corollaries will be given in the next section.

We start with an elementary lemma:

Lemma 2.2.11. Let $p \in M$ be a regular point for the $H$-action and $\Sigma$ the section through $p$. Then $\operatorname{ker}\left(d \mu_{p}\right)=J T_{p} \Sigma$.

Proof. First of all since $p$ is regular $T_{p} \Sigma$ is precisely the normal space of the orbit $H \cdot p$. The lemma follows then immediately from the standard fact that $\operatorname{ker}\left(d \mu_{p}\right)=\left(T_{p} H \cdot p\right)^{\perp_{\omega}}$, where $\perp_{\omega}$ denotes the orthogonal complement with respect to the Kähler form $\omega$ of $M$.

Now define

$$
M_{\mu}:=\{x \in M \mid \operatorname{dim} H \cdot \mu(x) \geq \operatorname{dim} H \cdot \mu(y) \text { for all } y \in M\},
$$

then in $[\mathrm{HuWu} 1990]$ it is proved that the set $M_{\mu}$ is open and that for all $x \in M_{\mu}$ the restricted momentum map

$$
\mu: H \cdot x \longrightarrow H \cdot \mu(x)
$$

is a principal $\left(S^{1}\right)^{r(x)}$-bundle, where $r(x):=\operatorname{dim}\left(T_{x} H \cdot x\right) \cap\left(T_{x} H \cdot x\right)^{\perp_{\omega}}$. For a polar action we have the following refinement:

Lemma 2.2.12. For any regular point $p \in M$ of the $H$-action $T:=H_{\mu(p)} / H_{p}$ is a torus with $\operatorname{dim} T=\operatorname{dim} \Sigma$.

Proof. Since the $H$-action is coisotropic we have $T_{p} H \cdot p^{\perp_{\omega}} \subset T_{p} H \cdot p$ and hence $r(p)=\operatorname{dim} \Sigma$ for all regular points $p$. Hence it is enough to show that every regular point of the $H$-action is contained in $M_{\mu}$. Since the set $M_{\mu}$ is open, it is enough to show that $\operatorname{dim} H \cdot \mu(p)$ is constant on the regular points of the $H$-action. For a regular point $p \in M$ we have

$$
T_{p} H \cdot p=J T_{p} \Sigma \oplus\left(T_{p} H \cdot p \cap J T_{p} H \cdot p\right)
$$

and $T_{p} H \cdot p \cap J T_{p} H \cdot p$ is isomorphic to $T_{\mu(p)} H \cdot \mu(p)$ via $d \mu_{p}$, which proves the lemma.

Now the torus $T=H_{\mu(p)} / H_{p}$ is at first an abstract torus and it is not clear how it acts on $M$. However on the Lie algebra level we have

$$
\mathfrak{h}_{\mu(p)}=\mathfrak{t} \oplus \mathfrak{h}_{p}
$$

with $\mathfrak{t}$ abelian. So in the following we mean by defintion that the notation $T=H_{\mu(p)} / H_{p}$ is a symbol which stands for the torus which is defined by $T=\exp \mathfrak{t}$. So $T$ is a subgroup of $H_{\mu(p)}$ and hence clearly acts on $M$. 
Lemma 2.2.13. Let $p \in M$ be a regular point and $T:=H_{\mu(p)} / H_{p}$. Then $T \cdot p$ describes the $\mu$-fiber through $p$, i. e. $\mu^{-1}(\mu(p))=T \cdot p$. Moreover we have $T_{p} T \cdot p=J T_{p} \Sigma$.

Proof. Viewing the restricted momentum map

$$
\mu: H \cdot p \rightarrow H \cdot \mu(p)
$$

as the homogeneous fibration

$$
H / H_{p} \rightarrow H / H_{\mu(p)}
$$

we see that $H_{\mu(p)} \cdot p$ is the fiber of the restricted momentum map. On the other hand we know that $\mu$ separates orbits since the action is coisotropic and hence $H_{\mu(p)} \cdot p$ is the fiber of the full momentum map. By the definition of $T$ it follows immediately that $T \cdot p=H_{\mu(p)} \cdot p$ : Let $g \in H_{\mu(p)}$. Since $H_{\mu(p)}$ is the centralizer of a torus it is connected and hence there exist $X \in \mathfrak{h}_{\mu(p)}$ with $\exp (X)=g$. Write $X=X_{1}+X_{2}$ with $X_{1} \in \mathfrak{t}$ and $X_{2} \in \mathfrak{h}_{p}$. Since $X_{1}$ and $X_{2}$ commute we have

$$
g p=\exp (X) p=\exp \left(X_{1}\right) \exp \left(X_{2}\right) p=\exp \left(X_{1}\right) p \in T \cdot p .
$$

The last claim is clear from the equality $\operatorname{ker}\left(d \mu_{p}\right)=J T_{p} \Sigma$.

Recall the distribution $\mathcal{D}$ defined by

$$
\mathcal{D}_{q}:=T_{q} \Sigma_{q} \oplus J T_{q} \Sigma_{q},
$$

where $q \in M_{r}$ and $\Sigma_{q}$ is the unique section through $q$. This gives us a well defined distribution on $M_{r}$ and by assumption this distribution is integrable. We have seen in Lemma 2.2.2 that the leaves are totally geodesic although they are not complete, since the distribution $\mathcal{D}$ is only defined on the regular set of the $H$-action. However since the ambient space is a symmetric space we can easily define a completion of a leaf $S_{p}$. Let $\Sigma$ be the section through $p$. Without loss of generality we can assume that $p$ is the origin of $M=G / K$. Let $\mathfrak{g}=\mathfrak{k} \oplus \mathfrak{p}$ be the corresponding Cartan decomposition and $\mathfrak{m} \subset \mathfrak{p}$ the tangent space of $\Sigma$. Then Lemma 2.2.2 implies that $\mathfrak{m} \oplus J \mathfrak{m}$ is a Lie triple system. Hence the following definition makes sense:

Definition 2.2.14. The complexification $\Sigma^{\mathbb{C}} \subset M$ of a section $\Sigma$ is defined by

$$
\Sigma^{\mathbb{C}}:=\exp _{p}\left(T_{p} \Sigma \oplus J T_{p} \Sigma\right)
$$

where $p \in \Sigma$ is any regular point.

The following proposition is then clear:

Proposition 2.2.15. The complexification $\Sigma^{\mathbb{C}}$ is a totally geodesic complex submanifold of $M$, which contains $\Sigma$. Moreover if $p \in \Sigma^{\mathbb{C}}$ is a regular point, then the leaf of $\mathcal{D}$ through $p$ is an open subset of $\Sigma^{\mathbb{C}}$. If $q \in \Sigma^{\mathbb{C}}$ is any regular point and $\Sigma_{q}$ is the section through $q$ then $\Sigma^{\mathbb{C}}$ is also the complexification of $\Sigma_{q}$ and we have $\Sigma^{\mathbb{C}}=\exp _{q}\left(T_{q} \Sigma_{q} \oplus J T_{q} \Sigma_{q}\right)$. 
Remark 2.2.16. The next proposition will show that alternatively one can define $\Sigma^{\mathbb{C}}:=\overline{T^{\mathbb{C}} \cdot p}$, where $p \in \Sigma$ is a regular point and $T=H_{\mu(p)} / H_{p}$. This is interesting in its own, since in general the closure of complex torus orbits can be highly singular.

Proposition 2.2.17. Let $p \in \Sigma$ be a regular point of the $H$-action on $M$ and $T=H_{\mu(p)} / H_{p}$. Then $T$ acts effectively, isometrically and in a Hamiltonian way on $\Sigma^{\mathbb{C}}$.

Proof. We will show that $H_{\mu(p)}$ acts on $\Sigma^{\mathbb{C}}$ and that $H_{p}$ is the kernel of this action. Remembering the homogeneous fibration

$$
H / H_{p} \rightarrow H / H_{\mu(p)}
$$

we see that $T_{p} H_{\mu(p)} \cdot p=J T_{p} \Sigma_{p}$ and hence it follows for any $g \in H_{\mu(p)}$ that

$$
T_{g p} H_{\mu(p)} \cdot p=d g\left(T_{p} H_{\mu(p)} \cdot p\right)=d g\left(J T_{p} \Sigma_{p}\right)=J T_{g p} \Sigma_{g p} \subset \mathcal{D}_{g p},
$$

which shows that $H_{\mu(p)} \cdot p \subset S_{p} \subset \Sigma^{\mathbb{C}}$. Now we can show that for an arbitrary point $x \in \Sigma^{\mathbb{C}}$ the orbit $H_{\mu(p)} \cdot x$ lies again in $\Sigma^{\mathbb{C}}$. Write $x=\exp _{p}(v+J w)$ with $v, w \in T_{p} \Sigma$. Using the fact that $H_{\mu(p)}$ acts isometrically we get for $g \in H_{\mu(p)}$ :

$$
g x=g \exp _{p}(v+J w)=\exp _{g p}(d g v+J d g w) \in \Sigma^{\mathbb{C}},
$$

since we already know that $g p \in \Sigma^{\mathbb{C}}$ and $d g v+J d g w \in T_{g p} \Sigma^{\mathbb{C}}$.

It is easy to see that the $T$-action on $\Sigma^{\mathbb{C}}$ is effective. We have to show that $H_{p}=\bigcap_{q \in \Sigma^{\mathbb{C}}}\left(H_{\mu(p)}\right)_{q}$. The inclusion " $\supset$ " follows from the trivial observation $\left(H_{\mu(p)}\right)_{p}=H_{p}$ while " $\subset$ " follows from the equality $\Sigma^{\mathbb{C}}=\exp _{p}\left(T_{p} \Sigma \oplus J T_{p} \Sigma\right)$ and the triviality of the slice representation of $H_{p}$ on $T_{p} \Sigma$. It is only left to prove that the action is Hamiltonian. But this is clear since the $T$-action on $M$ is Hamiltonian and $T$ leaves $\Sigma^{\mathbb{C}}$ invariant.

For the final proof we will need the following criterion for polarity:

Proposition 2.2.18 ([Go 2004]). Let $M=G / K$ be a symmetric space of compact type with a Riemannian metric induced from some $\operatorname{Ad}(G)$-invariant inner product on the Lie algebra $\mathfrak{g}$ of $G$. Consider a closed, connected subgroup $H \subset G$. $B y$ replacing $H$ by a conjugate, if necessary, we may assume that eK is a regular point. Write $\mathfrak{g}=\mathfrak{k} \oplus \mathfrak{p}$ for the Cartan decomposition, denote by $\mathfrak{h}$ the Lie algebra of $H$, and define $\mathfrak{m}=\mathfrak{p} \cap \mathfrak{h}^{\perp}$ (i. e. $\mathfrak{m}$ is the normal space to the $H$-orbit through $e K)$. Then the action of $H$ on $M$ is polar if and only if the following two conditions hold:

(i) $[[\mathfrak{m}, \mathfrak{m}], \mathfrak{m}] \subset \mathfrak{m}$

(ii) $[\mathfrak{m}, \mathfrak{m}] \perp \mathfrak{h}$.

Proof of Theorem 2.2.6. Since $\mathfrak{h} \supset \mathfrak{t}$ the polarity of the $T$-action is clear using the criterion of Gorodski. Since $T$ acts effectively and isometrically we have $T \subset \operatorname{Iso}\left(\Sigma^{\mathbb{C}}\right)$ and hence $\operatorname{rk}\left(\operatorname{Iso}\left(\Sigma^{\mathbb{C}}\right)\right) \geq \operatorname{dim} T=\operatorname{dim}_{\mathbb{C}} \Sigma^{\mathbb{C}}$. On the other hand 
we have the general fact that for an irreducible Hermitian symmetric space $N$ always $\operatorname{dim}_{\mathbb{C}} N \geq \operatorname{rk}(\operatorname{Iso}(N))$ and equality holds if and only if $N$ is a complex projective space. This shows $\Sigma^{\mathbb{C}}=\mathbb{P}_{n_{1}}(\mathbb{C}) \times \cdots \times \mathbb{P}_{n_{k}}(\mathbb{C})$. This argument was also used in the proof of Corollary 1.4. in [PoTh 2003]. 


\subsection{Applications of the Reduction Theorem}

\subsubsection{A hyperpolarity criterion}

Let us return to the momentum map $\mu: M \rightarrow \mathfrak{h}$ of the $H$-action. If $p \in M$ is a regular point of the $H$-action, then we have seen that the orbit T.p describes the momentum fiber through $p$. Interestingly these gives a criterion to distinguish a polar action from a hyperpolar one. More precisely:

Proposition 2.3.1. Let $M$ be an irreducible Hermitian symmetric space endowed with a complex integrable polar action of a compact connected Lie group $H$. The $H$-action is hyperpolar if and only if there exists a totally geodesic momentum fiber through a regular point of the $H$-action.

First we will prove a lemma which describes a splitting of the induced torus action on the complexified section.

Lemma 2.3.2. Let $p \in \Sigma^{\mathbb{C}}=\mathbb{P}_{n_{1}}(\mathbb{C}) \times \cdots \times \mathbb{P}_{n_{k}}(\mathbb{C})$ be a regular point of the $H$-action and $T:=H_{\mu(p)} / H_{p}$. Then $T$ splits into $T=\left(S^{1}\right)^{n_{1}} \times \cdots \times\left(S^{1}\right)^{n_{k}}$ such that the $T$-action is the product of polar actions of $\left(S^{1}\right)^{n_{i}}$ on $\mathbb{P}_{n_{i}}(\mathbb{C})$.

Proof. This is trivial. One only has to observe that $T$ is a maximal torus in the isometry group of $\Sigma^{\mathbb{C}}$.

The next lemma is also clear:

Lemma 2.3.3. Let $\Sigma^{\prime}$ be a section for the $H$-action which is contained in $\Sigma^{\mathbb{C}}$. Then $\Sigma^{\prime}$ is also a section for the T-action on $\Sigma^{\mathbb{C}}$, where $T=H_{\mu(p)} / H_{p}$ for any regular point $p \in \Sigma^{\mathbb{C}}$. Conversely any section for the $T$-action on $\Sigma^{\mathbb{C}}$ is also a section for the $H$-action on $M$.

Proof. Pick a regular point $x \in \Sigma^{\prime}$. Then there exists a section $\hat{\Sigma} \subset \Sigma^{\mathbb{C}}$ through $x$ for the $T$-action. This means there exists an element $t \in T$ with $t \cdot \Sigma=\hat{\Sigma}$. Since $x$ is a regular point and $T \subset H$ it follows that $\Sigma^{\prime}=\hat{\Sigma}$. The same argument proves the other direction.

In [HuWu 1990] it was shown that for all $p \in M_{\mu}$ the torus orbit $T \cdot p$ with $T:=H_{\mu(p)} / H_{p}$ describes the $\mu$-fiber through $p$. In our situation one can show that this torus describes not only the $\mu$-fiber through $p$, but also the fibers on a larger set. More precisely:

Proposition 2.3.4. Let $p \in \Sigma^{\mathbb{C}} \subset M$ be a regular point of the $H$-action and $T:=H_{\mu(p)} / H_{p}$. Then $T \cdot x$ is the $\mu$-fiber through $x$ for all regular $x \in \Sigma^{\mathbb{C}}$.

Proof. Let $x \in \Sigma^{\mathbb{C}} \subset M$ be a regular point of the $H$-action and $T^{\prime}:=H_{\mu(x)} / H_{x}$. If $\Sigma_{x}$ is the section for the $H$-action through $x$ then it follows from the lemma above that $\Sigma_{x}$ is contained in $\Sigma^{\mathbb{C}}$ and that it is a section for the $T$-action as well as for the $T^{\prime}$-action. It follows that the sections for the $T$-and the $T^{\prime}$-action are identical and hence the principal orbits of both actions coincide. 
Proof of Proposition 2.3.1. Let us first assume that there exists a totally geodesic $\mu$-fiber through a regular point $p \in M$ of the $H$-action. Let $\Sigma$ be the section through $p$ and $T:=H_{\mu(p)} / H_{p}$. Then we know that $T$ acts polarly on $\Sigma^{\mathbb{C}}$ with $\Sigma$ as a section. Let $N:=T \cdot p \subset \Sigma^{\mathbb{C}} \subset M$ denote the totally geodesic $\mu$-fiber through $p$. Pick two vectors $u, v \in T_{p} N$ and let $R^{L}$ denote the curvature tensor of a submanifold $L \subset M$. If $g, J$ denote the metric and the complex structure of $M$ we have

$$
\begin{aligned}
g\left(R^{\Sigma}(J u, J v) J v, J u\right) & =g\left(R^{\Sigma^{\mathbb{C}}}(J u, J v) J v, J u\right) \\
& =g\left(R^{\Sigma^{\mathbb{C}}}(u, v) v, u\right)=g\left(R^{N}(u, v) v, u\right)=0,
\end{aligned}
$$

since $N$ is a totally geodesic orbit of a torus and thus flat.

The converse direction follows from Lemma 2.3.2, since it shows that for a hyperpolar action the $T$-action splits into a product of $S^{1}$-action on a product of Riemann spheres.

\subsubsection{The rank one case}

Let $X$ be a complex projective space. Then any totally geodesic submanifold of $X$ is again a rank one space, hence it is known, that a section for a polar action on $X$ must be again a rank one space. Thorbergsson and Podestà have proven in [PoTh 1999] by classifying all polar actions on $X$ that a section is isometric to a real projective space. This will also follow from the following proposition:

Proposition 2.3.5. Let $M$ be an irreducible Hermitian symmetric space endowed with a complex integrable polar action of a compact connected Lie group $H$. Let $\Sigma$ be a section for the $H$-action. Then $\Sigma$ is isometric to a product of real projective spaces, i. e. $\Sigma=\mathbb{P}_{n_{1}}(\mathbb{R}) \times \cdots \times \mathbb{P}_{n_{k}}(\mathbb{R})$. The $H$-action is hyperpolar if and only if $n_{1}=\cdots=n_{k}=1$.

Proof. We have seen that for a section $\Sigma$ the complexification $\Sigma^{\mathbb{C}}$ is a product of complex projective spaces, i. e. $\Sigma^{\mathbb{C}}=\mathbb{P}_{n_{1}}(\mathbb{C}) \times \cdots \times \mathbb{P}_{n_{k}}(\mathbb{C})$. Let $p \in \Sigma$ be a regular point of the $H$-action and $T=H_{\mu(p)} / H_{p}$. We have seen in Lemma 2.3.2 that the $T$-action splits into $\left(S^{1}\right)^{n_{i}}$-actions on $\mathbb{P}_{n_{i}}(\mathbb{C})$. These actions are clearly conjugated to the action of the standard maximal torus in the isometry group of $\mathbb{P}_{n_{i}}(\mathbb{C})$, for which it is well known that it is polar with a section being isometric to $\mathbb{P}_{n_{i}}(\mathbb{R})$.

Proposition 2.3.6. Let $X$ be a complex projective space and $H$ a connected compact Lie group acting polarly on $X$. Then the action is complex integrable and a section is isometric to a real projective space.

Proof. We have to show that the distribution $\mathcal{D}$ is integrable. This follows from the fact that any complex subspace in the tangent space of $X$ is a Lie triple system (cf. [KoNo 1969] p. 277). It is then clear that we get a leaf by defining $S_{p}:=\exp _{p}\left(T_{p} \Sigma_{p} \oplus J T_{p} \Sigma_{p}\right) \cap M_{r}$. The claim follows then from the proposition above since $X$ is a rank one space. 


\subsubsection{Hermitian symmetric spaces of maximal rank}

In this section we will analyze polar actions on the Lagrangian Grassmannian of maximal isotropic planes in $\mathbb{C}^{2 n}$, i. e. the Hermitian symmetric space $M=G / K=\operatorname{Sp}(n) / \mathrm{U}(n)$. The special property of this space that makes it interesting is the fact that it is a space of maximal rank, i. e. the rank of $M$ is equal to the rank of its isometry group. This allows us to use some general results about toric varieties and torus actions on generalized flag manifolds. Recall the non-degenerate stratum $Z\left(T^{\mathbb{C}}\right)$ which was defined as the intersection of the big cells of all Borel subgroups containing a fixed maximal Torus $T^{\mathbb{C}}$.

Proposition 2.3.7. Let $H \times M \rightarrow M$ be a complex integrable hyperpolar action with maximal cohomogeneity ( $i$. e. $\operatorname{cohom}(H, M)=\mathrm{rk} G$ ) of a compact connected Lie group on $M$. Then every regular point $p \in M$ is contained in the nondegenerate stratum $Z\left(T^{\mathbb{C}}\right)$ of the complexification of the torus $T=H_{\mu(p)} / H_{p}$.

The first observation concerning this stratum was made by Gel'fand and Serganova in [GeSe 1987]. First they define the notion of a thin cell. Let $G^{\mathbb{C}}$ be a complex semisimple Lie group and $P \subset G^{\mathbb{C}}$ a parabolic subgroup. Then $G^{\mathbb{C}} / P$ is a generalized flag manifold. Let $B \subset G^{\mathbb{C}}$ be a Borel subgroup containing a fixed Cartan subgroup $T^{\mathbb{C}}$. The orbits of $B$ in $M$ are called the Schubert cells associated with $B$.

Definition 2.3.8. For every Borel subgroup $B$ containing $T^{\mathbb{C}}$ we choose precisely one Schubert cell. The intersection of all these chosen Schubert cells is called a thin cell, if it is not empty.

Remark 2.3.9. Let $W=N_{G}\left(T^{\mathbb{C}}\right) / T^{\mathbb{C}}$ denote the Weyl group. Then $W$ acts simply transitively on the set of all Borel subgroups containig $T^{\mathbb{C}}$. Let $B$ be a fixed Borel subgroup containing $T^{\mathbb{C}}$ and $w \in W$. Choosing for the Borel subgroup $w B w^{-1}$ the Schubert cell through $w P$ we see that $Z\left(T^{\mathbb{C}}\right)$ is a thin cell.

Let $T$ be a compact real form of $T^{\mathbb{C}}$ and $\mu_{T}: M \rightarrow \mathfrak{t}$ the momentum map for the action of $T$ on $M$. It is well known that the image of $\overline{T^{\mathbb{C}} \cdot p}$ under the momentum map is a convex polytope for all $p \in M$ [At 1982], [GuSt 1982]. We have the following result of Gel'fand and Serganova:

Theorem 2.3.10 (Theorem 1 in $\S 6$ of [GeSe 1987]). For two points $p, q \in M$ we have $\mu_{T}\left(\overline{T^{\mathbb{C}} \cdot p}\right)=\mu_{T}\left(\overline{T^{\mathbb{C}} \cdot q}\right)$ if and only if $p$ and $q$ lie in the same thin cell.

Remark 2.3.11. It is important to note that this theorem does not imply that $\overline{T^{\mathbb{C}} \cdot p}$ and $\overline{T^{\mathbb{C}} \cdot q}$ are isomorphic as toric varieties. The general theory of toric varieties is not directly applicable, because nothing is said about the normality of these varieties. This is a mistake Flaschka and Haine make in [FlHa 1991]. Their results only hold for those torus orbit closures in $G^{\mathbb{C}} / P$ which are normal. In particular contrary to what is claimed by them, there exist torus orbit closures in $Z\left(T^{\mathbb{C}}\right)$ which are not isomorphic.

However one can show that there exists an open subset of the non-degenerate stratum $Z\left(T^{\mathbb{C}}\right)$ such that all torus orbit closures are normal and isomorphic as 
toric varieties, as was done by Dabrowski [Da 1996]. Let us call this set $Y$ and torus orbit closures of elements in $Y$ generic. We will not go into the detailed description of this set, since we do not need it. We just briefly state the main result of [Da 1996]:

Theorem 2.3.12 (Theorem 3.2 of $\left[\right.$ Da 1996]). Let $x \in Y \subset G^{\mathbb{C}} / P$ and $X:=\overline{T^{\mathbb{C}} x}$. Then:

(i) $X$ is a normal variety.

(ii) The fan corresponding to $X$ consists of the cones

$$
C_{w}=-w \bigcup_{z \in W_{P}} z D, w \in W^{P}
$$

together with their faces. In particular the closure of any two orbits in $Y$ are isomorphic as $T^{\mathbb{C}}$-equivariant embeddings.

Here $D$ denotes the fundamental Weyl chamber with respect to a chosen basis of simple roots, $W_{P}$ is the subgroup of the Weyl group generated by the simple roots defining $P$ and $W^{P}$ is a fixed set of representatives of the coset space $W / W_{P}$.

The next step will be to identify the generic torus orbit type for $M=\operatorname{Sp}(n) / \mathrm{U}(n)$. As we have seen, this can be done by identifying the fan describing this toric variety.

Let $\Delta:=\left\{ \pm\left(e_{k} \pm e_{j}\right) \mid 1 \leq j, k \leq n, j \neq k\right\} \cup\left\{2 e_{i} \mid 1 \leq i \leq n\right\}$ be the root system of $\mathfrak{g}^{\mathbb{C}}=\mathfrak{s} p(n, \mathbb{C})$. Then a set of simple roots is given by $\pi=\left\{\alpha_{1}, \ldots, \alpha_{n}\right\}$ with $\alpha_{j}=e_{j}-e_{j+1}$ for $j=1, \ldots, n-1$ and $\alpha_{n}=2 e_{n}$. The parabolic subgroup $P \subset G^{\mathbb{C}}$ associated to $\pi_{P}:=\left\{\alpha_{1}, \ldots, \alpha_{n-1}\right\}$ realizes $M$ as the quotient $G^{\mathbb{C}} / P$. We realize this root system in an euclidean space $(E,(\cdot, \cdot))$ and denote by $W$ the Weyl group of it and by $W_{P}$ the subgroup of $W$ generated by reflections $s_{\alpha}$ with $s_{\alpha} \in \pi_{P}$. Let $D$ denote the fundamental Weyl chamber $\{v \in E \mid(v, \alpha) \geq 0$ for all $\alpha \in \pi\}$. We have the following lemma:

Lemma 2.3.13. $\bigcup_{z \in W_{p}} z D=\left\{v \in E \mid\left(v, e_{i}\right) \geq 0, i=1, \ldots, n\right\}$

Proof. Let $L$ denote the right hand side. The first step will be to show that $D \subset L$. So let $v \in D$ then $\left(e_{j}-e_{j+1}, v\right) \geq 0$ for all $j \in\{1, \ldots, n-1\}$ and $\left(e_{n}, v\right) \geq 0$. Setting $j=n-1$ and looking at the inequalities $\left(e_{n-1}-e_{n}, v\right) \geq 0$ and $\left(e_{n}, v\right) \geq 0$ we see that $\left(e_{n-1}, v\right) \geq 0$. Inductively we obtain $\left(e_{i}, v\right) \geq 0$ for all $i \in\{1, \ldots, n\}$, so $D \subset L$. Now we will show that the reflections $s_{\alpha}$ with $\alpha \in \pi_{P}$ leave $L$ invariant. Recall that the reflection $s_{\alpha}$ is given by $s_{\alpha}(w)=w-\frac{2(w, \alpha)}{(\alpha, \alpha)} \alpha$, we then obtain for $w \in L$ and $i \in\{1, \ldots, n-1\}, j \in\{1, \ldots, n\}$ :

$$
\left(s_{e_{i}-e_{i+1}}(w), e_{j}\right)=\left(w, s_{e_{i}-e_{i+1}}\left(e_{j}\right)\right)=\left\{\begin{array}{ll}
\left(w, e_{i+1}\right), & j=i \\
\left(w, e_{i}\right), & j=i+1 \\
\left(w, e_{j}\right), & j \notin\{i, i+1\}
\end{array}\right\} \geq 0,
$$

so $s_{e_{i}-e_{i+1}}(w) \in L$. Since the reflections $s_{\alpha}$ with $\alpha \in \pi_{P}$ generate $W_{P}$, we see that $W_{P}$ leaves $L$ invariant and hence $\bigcup_{z \in W_{P}} z D \subset L$. 
Now let $v \in L$. Then $v=\sum_{i=1}^{n} a_{i} e_{i}$ with $a_{i} \in \mathbb{R}_{\geq 0}$ and the reflection $s_{\alpha_{j}}=s_{e_{j}-e_{j+1}}$ acts on $v$ by permuting the coefficients $a_{j}$ and $a_{j+1}$. It is then clear that there exists numbers $i_{1}, \ldots, i_{k} \in\{1, \ldots, n-1\}$ sucht that $w:=s_{\alpha_{i_{1}}} \cdots s_{\alpha_{i_{k}}}(v)=\sum_{i=1}^{n} c_{i} e_{i}$ with $c_{1} \geq c_{2} \geq \ldots \geq c_{n} \geq 0$. It follows $\left(w, e_{i}-e_{i+1}\right)=c_{i}-c_{i+1} \geq 0$ for all $i \in\{1, \ldots, n-1\}$, i. e. $w \in D$ and hence $v \in z D$ with $z=\left(s_{\alpha_{i_{1}}} \cdots s_{\alpha_{i_{k}}}\right)^{-1}$. Since $z \in W_{P}$ the proof of the lemma is complete.

Proposition 2.3.14. The generic torus orbit type for $M$ is isomorphic to the product of $n$ complex projective lines.

Proof. Let $Q_{\varepsilon_{1}, \ldots, \varepsilon_{n}}:=\left\{\sum_{i=1}^{n} \varepsilon_{i} a_{i} e_{i} \mid a_{i} \in \mathbb{R}_{\geq 0}\right\}$ with $\varepsilon_{i} \in\{1,-1\}$ and $\mathcal{Q}$ the set of all such $Q_{\varepsilon_{1}, \ldots, \varepsilon_{n}}$. For the proof of this proposition it will be enough to show that

$$
\mathcal{Q}=\left\{-w \bigcup_{z \in W_{P}} z D \mid w \in W\right\} .
$$

First of all we note that thanks to Lemma 2.3.13 $\bigcup_{z \in W_{p}} z D=Q_{1, \ldots, 1}$. As already seen the reflection $s_{\alpha_{j}}$ with $1 \leq j \leq n-1$ acts on $v=\sum_{i=1}^{n} a_{i} e_{i}$ by permuting the coefficients $a_{j}$ and $a_{j+1}$. For the reflection $s_{\alpha_{n}}$ we have

$$
s_{\alpha_{n}}\left(e_{i}\right)=\left\{\begin{array}{rr}
e_{i} & i \neq n \\
-e_{n} & i=n
\end{array}\right.
$$

and hence $w \cdot Q_{1, \ldots, 1}=Q_{\varepsilon_{1}, \ldots, \varepsilon_{n}}$ for any $w \in W$ and some $\varepsilon_{i} \in\{1,-1\}$. It follows that the right hand side of $(2.3)$ is contained in the left hand side. Conversely we can create any $Q_{\varepsilon_{1}, \ldots, \varepsilon_{n}} \in \mathcal{Q}$ starting with $Q_{1, \ldots, 1}$ by successive applications of $s_{\alpha_{n}}$ (in order to create a minus sign) and the $s_{\alpha_{j}}$ with $1 \leq j \leq n-1$ (in order to bring the minus sign at the right place). Remembering Example 2.1.6 we see that $\mathcal{Q}$ describes indeed the fan of $\mathbb{P}_{1}(\mathbb{C})^{n}$.

We will now prove Proposition 2.3.7.

Proof of Proposition 2.3.7. Let $p \in M$ be a regular point of the $H$-action. It follows from the Reduction Theorem that the complexification of the section through $p$ is isomorphic to a product of complex projective lines and hence $\overline{T^{\mathbb{C}} \cdot p}=\mathbb{P}_{1}(\mathbb{C})^{n}$. Let $\mu_{T}: M \rightarrow \mathfrak{t}^{*}$ denote the momentum map for the $T$-action on $M$. Let $L^{T}$ denote the set of $T$-fixed points in $L$ for a subset $L \subset M$. Let $q \in Z\left(T^{\mathbb{C}}\right) \subset M$ be a point that realizes the generic torus orbit type of $M$. First we observe that the Euler characteristic of $M$ is $2^{n}$ (see for example [SCM 1997]) and hence both $\left(\overline{T^{\mathbb{C}} \cdot p}\right)^{T}$ and $\left(\overline{T^{\mathbb{C}} \cdot q}\right)^{T}$ contain all $T$-fixed points in $M$. Let $\operatorname{conv}(R)$ denote the convex hull of a subset $R \subset \mathfrak{t}$. Using the convexity results of [At 1982], [GuSt 1982], [Ki 1984] we obtain

$$
\mu_{T}\left(\overline{T^{\mathbb{C}} \cdot p}\right)=\operatorname{conv}\left(\mu\left(\left(\overline{T^{\mathbb{C}} \cdot p}\right)^{T}\right)\right)=\operatorname{conv}\left(M^{T}\right)=\operatorname{conv}\left(\mu\left(\left(\overline{T^{\mathbb{C}} \cdot q}\right)^{T}\right)\right)=\mu_{T}\left(\overline{T^{\mathbb{C}} \cdot q}\right),
$$

i. e. the momentum images of the torus orbit closures through $p$ and $q$ are identical and hence it follows with Theorem 2.3.10 that $p \in Z\left(T^{\mathbb{C}}\right)$ and the proposition is proven. 
Remark 2.3.15. Let $T_{K}^{\mathbb{C}}$ denote the complex torus that comes from the $K$ action on $M=G / K$ and let $T_{H}^{\mathbb{C}}$ be the complex torus for a polar action of a compact Lie group $H$. Then, up to conjugation, we have $T_{H}^{\mathbb{C}} \subset T_{K}^{\mathbb{C}}$ and it would be worthwhile to study how $T_{H}^{\mathbb{C}}$ sits inside $T_{K}^{\mathbb{C}}$.

\subsubsection{Two examples: $\operatorname{Sp}(2) / \mathrm{U}(2)$ and $\operatorname{Sp}(3) / \mathrm{U}(3)$}

As an application of the previous ideas we will prove the following result:

Proposition 2.3.16. $\quad$ (i) Any complex integrable polar action of a connected compact Lie group on $M_{2}:=\mathrm{Sp}(2) / \mathrm{U}(2)$ is hyperpolar.

(ii) Any complex integrable polar action of a connected compact Lie group on $M_{3}:=\mathrm{Sp}(3) / \mathrm{U}(3)$ with maximal cohomogeneity, i. e. cohomogeneity equal to three is hyperpolar.

Before we come to the proof we state the following lemma.

Lemma 2.3.17. Let $M=G / K$ be a Hermitian symmetric space realized as an adjoint orbit in $\mathfrak{g}$ and $T \subset G$ a maximal torus. Then all $T$-fixed points lie in the Lie algebra $\mathfrak{t}$ of $T$.

Proof. Let $p \in M \subset \mathfrak{g}$ be a $T$-fixed point. Then

$$
\{0\}=T_{p} T \cdot p=\{[\xi, p] \mid \xi \in \mathfrak{t}\},
$$

i. e. $p$ commutes with all of $\mathfrak{t}$. Since $\mathfrak{t}$ was assumed to be maximal the claim follows.

Proof of Proposition 2.3.16. Let $p \in M_{2}$ be a regular point of the $H$-action and $\Sigma$ the section through $p$. We have seen that $\Sigma^{\mathbb{C}}=\overline{T^{\mathbb{C}} \cdot p}$ for some torus $T \subset H$ and hence we know that $\operatorname{cohom}\left(H, M_{2}\right) \leq \mathrm{rk} \operatorname{Sp}(2)=2$. The $\operatorname{cohom}\left(H, M_{2}\right)=1$ case is trivial so let us assume that $\operatorname{cohom}\left(H, M_{2}\right)=2$. Then it follows from the Reduction Theorem that $\Sigma^{\mathbb{C}}=\mathbb{P}_{1}(\mathbb{C}) \times \mathbb{P}_{1}(\mathbb{C})$ or $\Sigma^{\mathbb{C}}=\mathbb{P}_{2}(\mathbb{C})$. We need to exclude the last case, so let us assume that $\Sigma^{\mathbb{C}}=\mathbb{P}_{2}(\mathbb{C})$. Let $\mu: M_{2} \rightarrow \mathfrak{h}$ be the momentum map for the $H$-action on $M_{2}$ and $T=H_{\mu(p)} / H_{p}$. Let $x \in M_{2}$ be a point that realizes the generic torus orbit type for $M_{2}$, i. e. $\overline{T^{\mathbb{C}} \cdot x}=\mathbb{P}_{1}(\mathbb{C}) \times \mathbb{P}_{1}(\mathbb{C})$. Recall that $\overline{T^{\mathbb{C}} \cdot x}$ contains all $T$-fixed points of the action of $T$ on $M_{2}$ since the Euler characteristic of $M_{2}$ is equal to four. Let $\mu_{T}: M_{2} \rightarrow \mathfrak{t}$ denote the momentum map for the $T$-action on $M_{2}$. We then have

$$
\mu_{T}\left(\Sigma^{\mathbb{C}}\right)=\operatorname{conv}\left(\mu_{T}\left(\left(\Sigma^{\mathbb{C}}\right)^{T}\right)\right) \subset \operatorname{conv}\left(\mu_{T}\left(M_{2}^{T}\right)\right)=\operatorname{conv}\left(\mu_{T}\left({\overline{T^{\mathbb{C}} \cdot x}}^{T}\right)\right)
$$

but this is a contradiction if we compare the momentum polytopes of $\mathbb{P}_{2}(\mathbb{C})$ and $\mathbb{P}_{1}(\mathbb{C}) \times \mathbb{P}_{1}(\mathbb{C})$, since the momentum polytope of $\mathbb{P}_{2}(\mathbb{C})$ is the convex hull of three equidistant $T$-fixed points, while the maximal cardinality of equidistant $T$-fixed points in $M_{2}$ is 2 . Note that thanks to Lemma 2.3.17 we can indeed say that the momentum polytopes in consideration are the convex hulls of certain $T$-fixed points. 

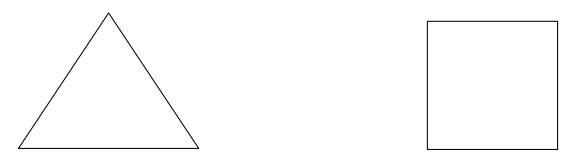

Figure 2.3: Momentum images of $\mathbb{P}_{2}(\mathbb{C})$ and $M_{2}$

Let us turn to the case of $M_{3}$. Let $\Sigma^{\mathbb{C}} \subset M_{3}$ be the complexification of a section. There are three possibilities for $\Sigma^{\mathbb{C}}$ :

$$
\Sigma^{\mathbb{C}}=\left\{\begin{array}{l}
\mathbb{P}_{3}(\mathbb{C}) \\
\mathbb{P}_{1}(\mathbb{C}) \times \mathbb{P}_{2}(\mathbb{C}) \\
\mathbb{P}_{1}(\mathbb{C}) \times \mathbb{P}_{1}(\mathbb{C}) \times \mathbb{P}_{1}(\mathbb{C})
\end{array}\right.
$$

The first case can be excluded by the same arguments as above: If $T=H_{\mu(p)} / H_{p}$ for a regular point $p \in \Sigma^{\mathbb{C}}$, then the four $T$-fixed points in $\Sigma^{\mathbb{C}}=\mathbb{P}_{3}(\mathbb{C})$ are all equidistant, but the maximal cardinality of equidistant $T$-fixed points in $M_{3}$ is three. So let us assume that $\Sigma^{\mathbb{C}}=\mathbb{P}_{1}(\mathbb{C}) \times \mathbb{P}_{2}(\mathbb{C})$. Then the $T$-action on $\Sigma^{\mathbb{C}}$ splits into a $S^{1}$-action on $\mathbb{P}_{1}(\mathbb{C})$ and a $S^{1} \times S^{1}$-action on $\mathbb{P}_{2}(\mathbb{C})$, so the momentum polytope of $\Sigma^{\mathbb{C}}$ is just the product of the polytopes of $\mathbb{P}_{1}(\mathbb{C})$ and $\mathbb{P}_{2}(\mathbb{C})$. It is easily seen that this polytope cannot be a subset of the momemtum polytope of $M_{3}$, which is as above equal to the momemtum polytope of a generic $\overline{T^{\mathbb{C}} \cdot x}=\mathbb{P}_{1}(\mathbb{C}) \times \mathbb{P}_{1}(\mathbb{C}) \times \mathbb{P}_{1}(\mathbb{C}) \subset M_{3}$
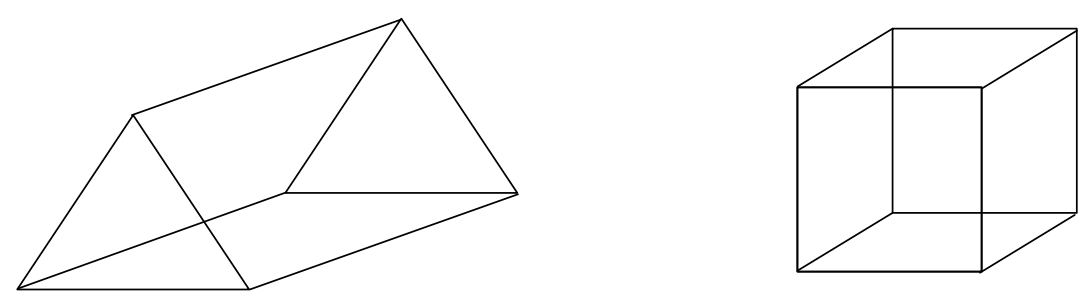

Figure 2.4: Momentum images of $\mathbb{P}_{1}(\mathbb{C}) \times \mathbb{P}_{2}(\mathbb{C})$ and $M_{3}$ 


\section{Chapter 3}

\section{Polar actions on Wolf spaces}

\subsection{Quaternion-Kähler manifolds}

In this section we briefly recall the basic results about quaternion-Kähler manifolds, mainly to fix notations. We omit all proofs that can be found easily in the literature.

Let $\mathbb{H}^{n}$ denote the quaternionic $n$-space. We can identify $\mathbb{H}^{n}$ with $\mathbb{R}^{4 n}$ such that the standard Euclidean inner product is given by $\langle u, v\rangle=\operatorname{Re}\left(v^{*} w\right)$, where * denotes transposing and quaternionically conjugating the entries. We can then define $\operatorname{Sp}(n):=\left\{A \in \mathrm{GL}(n, \mathbb{H}) \mid\langle A u, A v\rangle\right.$ for all $\left.u, v \in \mathbb{H}^{n}\right\}$. It is easily seen that the action of $\operatorname{Sp}(n)$ on $\mathbb{R}^{4 n}$ is isometric, so $\operatorname{Sp}(n) \subset \mathrm{SO}(4 n)$. However $\mathrm{Sp}(n)$ is not a maximal subgroup of $\mathrm{SO}(4 n)$ since it commutes with the right multiplication with $\operatorname{Sp}(1)$. One then defines $\operatorname{Sp}(n) \cdot \operatorname{Sp}(1):=\operatorname{Sp}(n) \times \operatorname{Sp}(1) / \mathbb{Z}_{2}$, where $\mathbb{Z}_{2}$ is generated by $(-I d,-1)$ and this group is indeed a maximal subgroup of $\mathrm{SO}(4 n)$ ([Gr 1965]). Now $\operatorname{Sp}(n) \cdot \operatorname{Sp}(1)$ appears also in the holonomy list of Berger so it is completely natural to define:

Definition 3.1.1. A 4n-dimensional $(n>1)$ Riemannian manifold is called quaternion-Kähler if its holonomy group is contained in $\operatorname{Sp}(n) \cdot \operatorname{Sp}(1)$. If $n=1$ we additionally require $M$ to be Einstein and self-dual.

There exist the following equivalent definition from a slightly different viewpoint:

Definition 3.1.2. Let $(M, g)$ be Riemannian manifold. We call $M$ a quaternionKähler manifold if there exists a parallel rank three subbundle $Q \subset \operatorname{End}(T M)$ which is locally spanned by three almost complex structures $I, J, K$ satisfying the usual quaternionic relations.

For a local section $I$ of $\mathrm{Q}$ one can then define a local two-form $\omega_{I}$ by setting $\omega_{I}(\cdot, \cdot):=g(I \cdot, \cdot)$. With this construction $Q$ can be identified with a subbundle of $\Lambda^{2} T^{*} M$. Analogously to the symplectic case we define:

Definition 3.1.3. Let $N$ be a submanifold of a quaternion-Kähler manifold $M$. We call $N$ a quaternion-coisotropic submanifold if and only if

$$
T_{p} N^{\perp \omega_{I}} \subset T_{p} N
$$


for all $p \in N$ and all sections $I$ of $Q$ locally defined around $p$. As in the symplectic case this is equivalent to saying that all normal spaces of $N$ are totally real with respect to $Q$. We call a Lie group action quaternion-coisotropic if and only if all principal orbits are quaternion-coisotropic.

An important tool in the analysis of quaternion-Kähler manifolds is the so called twistor space. Consider the unit-sphere subbundle $Z$ of the quaternionic bundle $Q \subset \operatorname{End}(T M)$. Let $I, J, K$ be a local basis of $Q$ around a point $p \in M$ as in the Definition 3.1.2. Then the $p$-fiber of $Z$ consists precisely of those linear combinations of $I, J, K$ which are complex structures on $T_{p} M$. We call $Z$ the twistor space of $M$. For a point $x \in Z_{p}:=\pi^{-1}(p)$ we denote by $I(x)$ the complex structure defined on $T_{p} M$. One can prove that for a general quaternion-Kähler manifold $M$ the twistor space $Z$ admits a natural complex structure, however for quaternion-Kähler spaces with positive Ricci curvature one has even more as was shown by Salamon ([Sa 1999]):

Theorem 3.1.4. Let $M$ be a quaternion-Kähler manifold with positive Ricci curvature and $\operatorname{dim} M>4$. Then its twistor space $Z$ admits a Kähler-Einstein metric with positive Ricci curvature such that the projection $\pi: Z \rightarrow M$ is a Riemannian submersion with totally geodesic fibers.

The natural complex structure $\mathcal{J}$ of $Z$ is pretty easy to describe. Let $\mathcal{H}$ (resp. $\mathcal{V}$ ) denote the horizontal (resp. vertical) subbundle of the Riemannian submersion $\pi: Z \rightarrow M$. A point $z \in Z$ corresponds to a complex structure $I(z)$ on $T_{\pi(z)} M$. This gives a complex structure on $\mathcal{H}_{z}$ via $\pi_{*}$. On $\mathcal{V}_{z}$ we get a complex structure by the canonical complex structure of the fiber $S^{2}$.

Now if $M$ is a Wolf space then its twistor space is even homogeneous. A Wolf space is a quaternion-Kähler symmetric space of compact type and it can be written as $M=G / K \cdot \operatorname{Sp}(1)$, where $G$ is a simple Lie group. The twistor space of $M$ is then given by $Z=G / K \cdot \mathrm{U}(1)$ for some $U(1) \subset \mathrm{Sp}(1)$ and the twistor fibration is just the homogeneous fibration

$$
\mathrm{Sp}(1) / \mathrm{U}(1) \hookrightarrow G / K \cdot \mathrm{U}(1) \rightarrow G / K \cdot \mathrm{Sp}(1) .
$$

In the next section the twistor space will be useful for us in the analysis of Kähler submanifolds of a quaternion-Kähler manifold. The following concepts are due to Alekseevsky and Marchiafava [AlMa 2001], [AlMa 2005]. In the following $M$ is assumed to be a positive quaternion-Kähler manifold, i. e. the Ricci curvature of $M$ is positive.

Definition 3.1.5. A submanifold $N$ of a quaternion-Kähler manifold $(M, g, Q)$ is called almost complex if there exists a section $J \in \Gamma\left(\left.Q\right|_{N}\right)$ such that

(i) $J^{2}=-I d$,

(ii) $J T N=T N$.

If $\left.J\right|_{T N}$ is integrable then $(N, J)$ is a complex manifold. 
Definition 3.1.6. An almost complex submanifold $(N, J)$ of $(M, g, Q)$ is called supercomplex if

$$
\nabla_{J X} J-J \nabla_{X} J=0 \text { for all } X \in T N,
$$

where $\nabla$ is of course the Levi-Civita connection of $(M, g)$.

We will need the following two results:

Proposition 3.1.7 (Theorem 1.1 of [AlMa 2001]). Let $(N, J)$ be an almost complex submanifold of $M$ with $\operatorname{dim} N>2$. Then $N$ is complex if and only if it is supercomplex.

Remark 3.1.8. Note that an almost complex manifold of dimension two is automatically complex.

Proposition 3.1.9 (Corollary 4.6 of [AlMa 2005]). Let $X$ be a complex submanifold of the twistor space $Z$ of $M$ and assume that the restricted twistor projection $\pi: X \rightarrow Y:=\pi(X)$ is a diffeomorphism. Then $Y$ is a supercomplex manifold of $M$ and any supercomplex manifold of $M$ has such form. 


\subsection{Polar actions on Wolf spaces}

Let $(X, \omega)$ be a compact simply-connected irreducible homogeneous Kähler manifold and $H$ a compact connected Lie group acting polarly on $M$. Then it is known that the $H$-action is coisotropic ([PoTh 2002]). In other words the sections for the $H$-action are totally real with respect to the complex structure $J$, i. e. $T_{p} \Sigma \perp J T_{p} \Sigma$ for all sections $\Sigma \subset M$ and all points $p \in \Sigma$.

Now let $M$ be a Wolf space endowed with a polar action of a compact connected Lie group $H$. Although there does not exist a complex structure on $M$, we can still ask a similar question: Let $Q \subset \operatorname{End}(T M)$ be the rank three vector bundle defining the quaternionic structure. Are the sections of the $H$-action totally real with respect to $Q$ ? More precisely we would like to prove:

Theorem 3.2.1. Let $M$ be a Wolf space and $H$ a connected compact Lie group acting polarly on $M$. Then the $H$-action is quaternion-coisotropic, $i$. e. the sections are totally real with respect to $Q$.

Let us briefly recall the proof of the Kähler case ([PoTh 2002]). There the key observation is, that the $H$-action on $X$ admits a complex orbit. Hence there exists a polar slice representation on a complex vector space and basically Dadoks Theorem allows to show that the sections for the slice representation are totally real. So the first idea in the quaternion-Kähler case would be to search for a quaternionic orbit of the $H$-action. We cannot guarantee the existence of a quaternionic orbit, however we will prove the following result:

Theorem 3.2.2. Let $M$ be a Wolf space and $H$ a compact connected Lie group acting isometrically on $M$. Then the $H$-action admits at least one of the following orbits:

(i) a complex orbit,

(ii) a totally geodesic quaternionic orbit,

(iii) a $\mathbb{Z}_{2}$-quotient of a complex orbit in the twistor space $Z$ of $M$.

The key idea for the proof of this result is to lift the $H$-action to a holomorphic action on the twistor space $Z \rightarrow M$. There one can find a complex orbit (cf. the appendix) and project this orbit down to $M$ to find a good candidate for a quaternionic orbit.

Lemma 3.2.3. Let $M$ be a Wolf space and $\pi: Z \rightarrow M$ the twistor fibration. Let $H$ be a compact connected Lie group acting isometrically on $M$. Then the $H$-action lifts to an equivariant action on $Z$ and there exists a complex $H$-orbit in $Z$.

Proof. Let $G$ denote the identity component of the isometry group of $M$. Then an element $g \in G$ acts on $Z$ by sending a complex structure $J$ to the complex structure $\tilde{g}(J)=g_{*} J g_{*}^{-1}$. Nitta and Takeuchi [NiTa 1987] have proven that the correspondence $g \leftrightarrow \tilde{g}$ realizes $G$ as a real form of the identity component of holomorphic automorphisms of the contact structure on $Z$ (see also 
[PoSa 1991]). This means that $H^{\mathbb{C}}$ acts on $Z$ and if $H^{\mathbb{C}}=H \cdot S$ is the Iwasawa decomposition of $H^{\mathbb{C}}$ then $S$ has a fixed point $z$ in $Z$ by the Borel fixed point theorem. The $H$-orbit through $z$ is complex since $H^{\mathbb{C}} \cdot z=H \cdot S \cdot z=H \cdot s$. Actually this is only a rough sketch of the proof, since $H$ need not be semisimple and hence the Iwasawa decomposition is not defined. However this is only a technical problem since one can pass to a certain cover of $H$. For the full details confer the appendix.

Lemma 3.2.4. Let $M$ be a Wolf space and $\pi: Z \rightarrow M$ the twistor fibration. Assume that a compact connected Lie group $H$ acts equivariantly with respect to $\pi$ on $M$ and $Z$. If for $z \in Z$ the orbit $H \cdot z$ is complex then all complex structures in the fiber $\pi^{-1}(\pi(z))$ leave the tangent space in $\pi(z)$ of $H \cdot \pi(z)$ invariant.

Proof. Let $X \in T_{\pi(z)} H \cdot \pi(z)=\pi_{*} T_{z} H \cdot z$. Choose a vector $X^{\prime} \in T_{z} H \cdot z$ with $X=\pi_{*} X^{\prime}$ and decompose $X^{\prime}$ into its horizontal and vertical part, i. e. $X^{\prime}=X_{1}^{\prime}+X_{2}^{\prime}$ with $X_{1}^{\prime} \in \mathcal{V}_{z}$ and $X_{2}^{\prime} \in \mathcal{H}_{z}$. Let $x \in \pi^{-1}(\pi(z))$. We then have

$$
I(x) X=I(x) \pi_{*} X^{\prime}=I(x) \pi_{*}\left(X_{1}^{\prime}+X_{2}^{\prime}\right)=I(x) \pi_{*} X_{2}^{\prime}=\pi_{*} \mathcal{J} X_{2}^{\prime}=\pi_{*} \mathcal{J} X^{\prime}
$$

and hence $I(x) X \in T_{\pi(z)} H \cdot \pi(z)$, since $\mathcal{J} X^{\prime} \in T_{z} H \cdot z$.

We now come to the proof of Theorem 3.2.2:

Proof of Theorem 3.2.2. Let $\pi: Z \rightarrow M$ be the twistor fibration. We have seen that the $H$-action lifts to an action on $Z$ and that there exists a point $z_{0} \in Z$ such that $H \cdot z_{0}$ is complex. We set $p_{0}:=\pi\left(z_{0}\right)$ and we will prove that the orbit $H \cdot p_{0} \subset M$ is of the required type. Let us first assume that the restriction $\hat{\pi}: H \cdot z_{0} \rightarrow H \cdot p_{0}$ of $\pi$ to the orbit in $Z$ is a diffeomorphism. Then Corollary 4.6 of [AlMa 2005] shows that $H \cdot p_{0}$ is a supercomplex submanifold of $M$ and Theorem 1.1 of [AlMa 2001] implies that $H \cdot p_{0}$ is a complex submanifold of $M$.

So let us now assume that the restriction of $\pi$ is not a diffeomorphism. There are two possibilities for $\hat{\pi}$ : Either there exists a point $x \in H \cdot z_{0}$ such that the differential of $\hat{\pi}$ in this point has not full rank or $\hat{\pi}$ is not injective. Let us first assume the former one. Without loss of generality we can assume that $x=z_{0}$. We will prove that in this case $H \cdot p_{0}$ is a quaternionic orbit. Let $v \in \operatorname{ker} d \hat{\pi}_{z_{0}}$ be a non-trivial vector. Since the orbit $H \cdot z_{0}$ is complex we have $\mathcal{J} v \in T_{z_{0}} H \cdot z_{0}$. Then

$$
d \hat{\pi}_{z_{0}} \mathcal{J} v=I\left(z_{0}\right) d \hat{\pi}_{z_{0}} v=0
$$

shows that the vertical part $\mathcal{V}_{z_{0}}$ of $T_{z_{0}} Z$ is contained in ker $d \hat{\pi}_{z_{0}}$. Since $\pi$ is equivariant with respect to the $H$-action we even have $\mathcal{V}_{z} \subset T_{z} H \cdot z_{0}$ for all $z \in H \cdot z_{0}$. This shows that for all $z \in H \cdot z_{0}$ the $\pi$-fiber thorugh $z$ is contained in $H \cdot z_{0}$. Then Lemma 3.2.4 immediately shows that the orbit $H \cdot p_{0}$ is quaternionic. The fact that $H \cdot p_{0}$ is totally geodesic follows from the well known fact that a quaternionic submanifold is automatically totally geodesic (cf. [Gr 1965]). Now we come to the last case: The restriction $\hat{\pi}$ is not a diffeomorphism but its differential has full rank everywhere. This means that $\hat{\pi}$ is not injective, i. e. there exists a point $x \in H \cdot z_{0}$ such that the cardinality of the set $\hat{\pi}^{-1}(\hat{\pi}(x)) \cap H \cdot z_{0}$ is bigger than one. Assume that there exist two points 
$z_{1}, z_{2} \in \hat{\pi}^{-1}(\hat{\pi}(x)) \cap H \cdot z_{0}$ such that the complex structures $I\left(z_{1}\right)$ and $I\left(z_{2}\right)$ are linearly indedpendent. Then $I\left(z_{1}\right) I\left(z_{2}\right)$ is a third linearly independent complex structure and since all these complex structures leave $T_{p_{0}} H \cdot p_{0}$ invariant it follows that the orbit $H \cdot p_{0}$ is quaternionic. Clearly there exist two linearly independent complex structures in $\hat{\pi}^{-1}(\hat{\pi}(x)) \cap H \cdot z_{0}$ as soon as the cardinality of this set is greater or equal to three. So let us assume that the cardinality is precisely two. Moreover we can assume that the two distinct points in $\hat{\pi}^{-1}(\hat{\pi}(x)) \cap H \cdot z_{0}$ are antipodal to each other because otherwise the corresponding complex structures would be linearly independent. It follows that $\hat{\pi}: H \cdot z_{0} \rightarrow H \cdot p_{0}$ is a two-fold covering since $\hat{\pi}$ is a surjective local diffeomorphism and $H \cdot z_{0}$ is a complete connected manifold.

As long as this proposition cannot be improved a direct generalization of the proof of [PoTh 2002] is not possible. However Theorem 3.2.1 can be proved if one uses the following recent classification result of Kollross:

Theorem 3.2.5. Let $M$ be a compact symmetric space of rank greater than one whose isometry group $G$ is simple. Let $H \subset G$ be a closed connected nontrivial subgroup acting polarly on $M$. Then the action of $H$ on $M$ is hyperpolar, that is, the sections are flat in the induced metric. Moreover, the sections are embedded submanifolds.

The higher rank case follows then from the next Lemma.

Lemma 3.2.6. Let $H$ be a connected compact Lie group acting hyperpolarly on a Wolf space $M=G / K$. Then the sections are totally real with respect to the quaternionic structure.

Proof. Let $\mathfrak{g}=\mathfrak{k} \oplus \mathfrak{p}$ denote the corresponding Cartan decomposition and $\mathfrak{s p}(1) \subset \mathfrak{k}$ the subalgebra defining the quaternionic structure, which means there exist three generators $z_{1}, z_{2}, z_{3} \in \mathfrak{s p}(1)$ such that

$$
\operatorname{ad}_{z_{i}}: \mathfrak{p} \rightarrow \mathfrak{p} \quad i=1,2,3
$$

are complex structures in the origin defining the quaternionic structure. Let $\Sigma \subset M$ be a section for the $H$-action and $\mathfrak{m} \subset \mathfrak{p}$ its tangent space in the origin. It follows

$$
\left\langle\mathfrak{m}, \operatorname{ad}_{z_{i}}(\mathfrak{m})\right\rangle=\left\langle\mathfrak{m},\left[z_{i}, \mathfrak{m}\right]\right\rangle=\left\langle z_{i},[\mathfrak{m}, \mathfrak{m}]\right\rangle=0
$$

which proves the lemma.

The proof of Theorem 3.2.1 follows then from the following proposition:

Proposition 3.2.7. Let $M$ be a rank one quaternion-Kähler symmetric space, i. e. $M=\mathbb{P}_{n}(\mathbb{H})$ and $H$ a connected compact Lie group acting polarly on $M$. Then the sections of the $H$-actions are totally real with respect to the quaternionic structure.

Before we come to proof we cite a result of Podestà and Thorbergsson [PoTh 1999] which we will need: 
Theorem 3.2.8. Let $H$ be a connected compact Lie group acting polarly on $\mathbb{P}_{n}(\mathbb{H})(n>1)$. Then the $H$-action is orbit equivalent to an action induced by the isotropy representation of $(U, K)=\Pi_{i=1}^{k}\left(U_{i}, K_{i}\right)$ such that the factors $U_{i} / K_{i}$ are quaternion-Kähler symmetric spaces, where at least $k-1$ of them have rank one.

Proof of Proposition 3.2.7. We briefly recall how the quaternionic structure on $M=\mathbb{P}_{n}(\mathbb{H})$ can be constructed from the one of $\mathbb{H}^{n+1}$. We write $M$ as the quotient $\left(\mathbb{H}^{n+1} \backslash\{0\}\right) / \mathbb{H}^{*}$ with respect to the natural right multiplication of $\mathbb{H}^{*}$ on $\mathbb{H}^{n+1} \backslash\{0\}$. We can identify $\mathbb{H}^{n+1}$ with $\mathbb{R}^{4 n+4}$, so let $\langle\cdot, \cdot\rangle$ denote the standard inner product. We then get an $\mathrm{Sp}(1)$-principal bundle

$$
\operatorname{Sp}(1) \hookrightarrow S^{4 n+3} \stackrel{\pi}{\rightarrow} \mathbb{P}_{n}(\mathbb{H}) .
$$

Let $p \in \mathbb{P}_{n}(\mathbb{H})$ be an arbitrary point and $z \in \pi^{-1}(p)$. The quaternionic structure of $\mathbb{P}_{n}(\mathbb{H})$ in the point $p$, i. e. on the tangent space $T_{p} \mathbb{P}_{n}(\mathbb{H})$ can be constructed as follows: Let $\mathcal{H}_{z} \subset T_{z} S^{4 n+3}$ denote the horizontal space with respect to $\langle\cdot, \cdot\rangle$, i. e.

$$
\mathcal{H}_{z}:=\operatorname{ker} d \pi_{z}^{\perp}=\left\{w \in \mathbb{H}^{n+1} \mid 0=\langle z, w\rangle=\langle z i, w\rangle=\langle z j, w\rangle=\langle z k, w\rangle\right\},
$$

then $\mathcal{H}_{z}$ is invariant under right multiplication with $i, j, k$ and this gives via the isomorphism $d \pi_{z}: \mathcal{H}_{z} \rightarrow T_{p} \mathbb{P}_{n}(\mathbb{H})$ the desired quaternionic structure: For example one defines $I: T_{p} \mathbb{P}_{n}(\mathbb{H}) \rightarrow T_{p} \mathbb{P}_{n}(\mathbb{H})$ by setting for $X \in T_{p} \mathbb{P}_{n}(\mathbb{H})$ : $I X:=d \pi_{z}(\hat{X} i)$, where $\hat{X} \in \mathcal{H}_{z}$ with $d \pi_{z} \hat{X}=X$.

Consider first the case $\mathbb{P}_{n}(\mathbb{H})$ with $n>1$. Let $\Sigma \subset \mathbb{P}_{n}(\mathbb{H})$ be a section for the $H$-action. The above cited result of Podestà and Thorbergsson implies that there exist a symmetric pair $(U, K)$ such that we can assume that $\Sigma$ is the projectivization $\mathbb{P}(\mathfrak{a})$ of a linear section $\mathfrak{a} \subset \mathfrak{p} \cong \mathbb{H}^{n+1}$ for the $K$-action on $\mathfrak{p}$, where $\mathfrak{u}=\mathfrak{k} \oplus \mathfrak{p}$ is the corresponding Cartan decomposition. Now we decompose $\mathfrak{u}$ into its irreducible components $\mathfrak{u}=\sum_{l=1}^{m} \mathfrak{u}_{i}$ and consider the corresponding Cartan decompositions $\mathfrak{u}_{l}=\mathfrak{k}_{l} \oplus \mathfrak{p}_{l}$. The complex structures $I, J, K: \mathfrak{p} \rightarrow \mathfrak{p}$ defining the quaternionic structure decompose into $I_{l}, J_{l}, K_{l}: \mathfrak{p}_{l} \rightarrow \mathfrak{p}_{l}$ such that each of them has the form $\operatorname{ad}_{w}$ for some $w \in \mathfrak{k}_{l}$. Let $z \in \mathfrak{a} \cap S^{4 n+3} \subset \mathfrak{p} \cong \mathbb{H}^{n+1}$. We claim that $z^{\perp} \cap \mathfrak{a} \subset \mathcal{H}_{z}$. Let $x \in z^{\perp} \cap \mathfrak{a}$. We will have to prove

$$
0=\langle I z, x\rangle=\langle J z, x\rangle=\langle K z, x\rangle,
$$

but this follows easily: Decompose $\mathfrak{a}=\sum_{l=1}^{m} \mathfrak{a}_{l}$ such that each $\mathfrak{a}_{l}$ is a section for the polar action of $K_{l}$ on $\mathfrak{p}_{l}$ and write

$$
x=\sum_{l=1}^{m} x_{l}, \quad z=\sum_{l=1}^{m} z_{l}, \quad I_{l}=\operatorname{ad}_{w_{l}} \text { with } x_{l}, z_{l} \in \mathfrak{a}_{l}, w_{l} \in \mathfrak{k}_{l} .
$$

We then get

$$
\langle I z, x\rangle=\left\langle I \sum_{l=1}^{m} z_{l}, \sum_{r=1}^{m} x_{r}\right\rangle=\sum_{l=1}^{m}\left\langle I_{l} z_{l}, x_{l}\right\rangle=\sum_{l=1}^{m}\left\langle\left[w_{l}, z\right], x\right\rangle=0 .
$$


The same calculations with $J$ and $K$ prove the claim.

Now let $X, Y \in T_{\pi(z)} \Sigma=T_{\pi(z)} \mathbb{P}(\mathfrak{a})$ and $X^{\prime}, Y^{\prime} \in z^{\perp} \cap \mathfrak{a} \subset \mathcal{H}_{z}$ with $d \pi_{z} X^{\prime}=X$ and $d \pi_{z} Y^{\prime}=Y$. Let $g$ denote the Riemannian metric of $\mathbb{P}_{n}(\mathbb{H})$ and $\tilde{I}$ the complex structure on $T_{\pi(z)} \mathbb{P}_{n}(\mathbb{H})$ defined by $I$. We get

$$
g(\tilde{I} X, Y)=g\left(d \pi_{z} I X^{\prime}, d \pi_{z} Y^{\prime}\right)=\left\langle I X^{\prime}, Y^{\prime}\right\rangle=0
$$

i. e. $T_{\pi(z)} \Sigma$ is totally real with respect to $\tilde{I}$. Of course the argument carries over to the other two complex structures.

Now assume $n=1$, i. e. $M \cong S^{4}$. Polar actions on spheres are restrictions of polar representations. If follows from Dadoks result [Dad 1985] that all polar actions on $S^{4}$ have cohomogeneity one and hence they are trivially quaternioncoisotropic. 


\section{Appendix}

In this appendix we supplement the details of the proof for the existence of a complex orbit for an isometric action on a compact homogeneous simplyconnected Kähler manifold. This fact was used in the proof of Theorem 3.2.2.

Theorem. Let $G$ be a compact connected real Lie group acting isometrically on a compact simply-connected homogeneous Kähler manifold $M$. Then $G$ has a complex orbit.

Proof. Let $G_{s} \times T^{m}$ be a covering of $G$ with $G_{s}$ a compact and semisimple Lie group and $T$ a torus. Let $\tilde{G}_{s}$ be the universal covering of $G_{s}$. We note that $\tilde{G}_{s}$ is also semisimple since $G_{s}$ and $\tilde{G}_{s}$ are locally isomorphic. The group $H:=\tilde{G}_{s} \times \mathbb{R}^{m}$ is a covering of $G_{s} \times T^{m}$ and hence also one of $G$. Let $\pi$ denote the covering map. We can define an action of $H$ on $M$ by setting $h \cdot p:=\pi(h) \cdot p$ for $h \in H, p \in M$. We clearly have $H \cdot p=G \cdot p$ for all $p \in M$. The action of $H$ on $M$ is isometric and the map $g: M \rightarrow M$ is holomorphic for all $g \in H$. Both $\tilde{G}_{s}$ and $\mathbb{R}^{m}$ are simply-connected and one easily sees that $H^{\mathbb{C}}=\tilde{G}_{s}^{\mathbb{C}} \times \mathbb{C}^{m}$. Since $\tilde{G}_{s}$ is compact we have $H \subset H^{\mathbb{C}}$ and there exists an action of the universal complexification $H^{\mathbb{C}}$ on $M$, whose restriction to $H$ is the original action. We are done if we can show that there exists a point $p \in M$ with $H^{\mathbb{C}} \cdot p=H \cdot p$. Since $\tilde{G}_{s}$ is compact, we see that $\tilde{\mathfrak{g}}_{s}$ is a real form of the Lie algebra $\tilde{\mathfrak{g}}_{s}^{\mathbb{C}}$ of $\tilde{G}_{s}^{\mathbb{C}}$, i. e. $\tilde{\mathfrak{g}}_{s}^{\mathbb{C}}=\tilde{\mathfrak{g}}_{s} \oplus i \tilde{\mathfrak{g}}_{s}$ is a Cartan decomposition of $\tilde{\mathfrak{g}}_{s}^{\mathbb{C}}$. Since $\tilde{G}_{s}^{\mathbb{C}}$ is semisimple, this implies that there exists a solvable Lie subalgebra $\mathfrak{s}$ such that $\tilde{\mathfrak{g}}_{s}^{\mathbb{C}}=\tilde{\mathfrak{g}}_{s} \oplus \mathfrak{s}$ is an Iwasawa decomposition of $\tilde{\mathfrak{g}}_{s}^{\mathbb{C}}$. This corresponds to an Iwasawa decomposition on the group level $\tilde{G}_{s}^{\mathbb{C}}=\tilde{G}_{s} S$, where $S$ is the simply connected Lie group with Lie algebra $\mathfrak{s}$. This leads to a decomposition of $H^{\mathbb{C}}$ :

$$
H^{\mathbb{C}}=\tilde{G}_{s}^{\mathbb{C}} \times \mathbb{C}^{m}=\tilde{G}_{s} S \times \mathbb{C}^{m} .
$$

Let $L$ denote the identity component of the group of holomorphic transformations of $M$. Then $L$ also acts transitively on $M$ and since the action of $H^{\mathbb{C}}$ can be restricted to $S \times \mathbb{C}^{m}$, we have $S \times \mathbb{C}^{m} \subset L$. We write $M=L / P$, where $P$ is the isotropy group of a point $p_{0} \in M$. Let $\mathfrak{l}$ denote the Lie algebra of $L$ and $\mathfrak{p}$ that of $P$. We can regard $\mathfrak{p}$ as a point in $G_{k}(\mathfrak{l})$, where $G_{k}(\mathfrak{l})$ denotes the Grassmannian of complex $k$-planes in $\mathfrak{l}$ and $k=\operatorname{dim}_{\mathbb{C}} \mathfrak{p}$. The Lie group $L$ acts on $\mathfrak{l}$ trough the adjoint representation and this gives us an action of $L$ on $G_{k}(\mathfrak{l})$ :

$$
L \times G_{k}(\mathfrak{l}) \rightarrow G_{k}(\mathfrak{l}), \quad(g, E) \mapsto \operatorname{Ad}(g) E
$$

and the orbit through $\mathfrak{p}$ is isomorphic to $L / L_{\mathfrak{p}}$. We claim $L_{\mathfrak{p}}=N(P)$, where $N(P)$ is the normalisator of $P$ in $L$, i. e. $N(P)=\left\{g \in L \mid g P g^{-1}=P\right\}$. 
Let $g \in N(P), v \in \mathfrak{p}$. Then there exists a path $\gamma$ in $P$ with $\gamma(0)=e$ and $\dot{\gamma}(0)=v$. If $c_{g}$ denotes the conjugation with $g$, then $\left(c_{g} \circ \gamma\right)(t)$ is a path in $P$ and $\operatorname{Ad}(g) v=\left.\frac{d}{d t}\right|_{t=0}\left(c_{g} \circ \gamma\right)(t) \in \mathfrak{p}$, so $g \in L_{\mathfrak{p}}$. For the converse inclusion take elements $g \in L_{\mathfrak{p}}$ and $p \in P$. Since $P$ is connected, there exist $X_{1}, \ldots, X_{n} \in \mathfrak{p}$ with $p=\exp \left(X_{1}\right) \cdot \ldots \cdot \exp \left(X_{n}\right)$ and

$$
g p g^{-1}=g \exp \left(X_{1}\right) g^{-1} g \exp \left(X_{2}\right) g^{-1} g \cdot \ldots \cdot g^{-1} g \exp \left(X_{n}\right) g^{-1} .
$$

This implies that it is enough to show that $g \exp \left(X_{i}\right) g^{-1} \in P$ for all $i=1, \ldots, n$. We have $\operatorname{Ad}(g) X_{i} \in \mathfrak{p}$ and so

$$
g \exp \left(X_{i}\right) g^{-1}=c_{g}\left(\exp \left(X_{i}\right)\right)=\exp \left(\operatorname{Ad}(g) X_{i}\right) \in P
$$

which proves our claim.

Since $P$ is a parabolic group we have $N(P)=P$. This implies that $M$ can be realized as an adjoint orbit in the Grassmannian $G_{k}(\mathfrak{l})$ :

$$
M=L / P=L / N(P)=L / L_{\mathfrak{p}}=\operatorname{Ad}(L) \mathfrak{p}
$$

We use the Plücker embedding to embed $G_{k}(\mathfrak{l})$ into the projective space $\mathbb{P}\left(\Lambda^{k} \mathfrak{l}\right)$, so $M$ is a projective subvariety of $\mathbb{P}\left(\Lambda^{k} \mathfrak{l}\right)$. We have the inclusions $\operatorname{Ad}(L) \subset \operatorname{GL}(\mathfrak{l}) \subset \operatorname{GL}\left(\Lambda^{k} \mathfrak{l}\right)$, where the last inclusion is realized via the map $e_{1} \wedge \ldots \wedge e_{n} \mapsto A e_{1} \wedge \ldots \wedge A e_{n}$ for $A \in \mathrm{GL}(\mathfrak{l})$. This means that $V=\Lambda^{k} \mathfrak{l}$ is a representation space of $L$ and $L$ acts on $\mathbb{P}\left(\Lambda^{k} \mathfrak{l}\right)$. The chosen embeddings of $\operatorname{Ad}(L)$ and $M$ are in the following sense compatible: The diagram

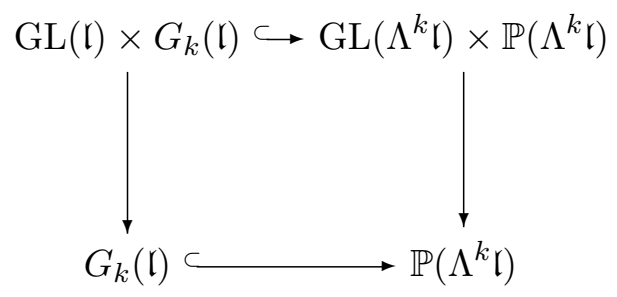

commutes, where the vertical arrows denote the group actions.

The action of $S \times \mathbb{C}^{m}$ on $\operatorname{Ad}(L) \mathfrak{p}$ that corresponds to the original action of $S \times \mathbb{C}^{m}$ on $M$ is just the adjoint action of $S \times \mathbb{C}^{m}$ : The identification map $\phi: M=L \cdot p_{0} \rightarrow \operatorname{Ad}(L) \mathfrak{p}$ is given by $\phi\left(f p_{0}\right)=\operatorname{Ad}(f)(\mathfrak{p})$ and the diagram

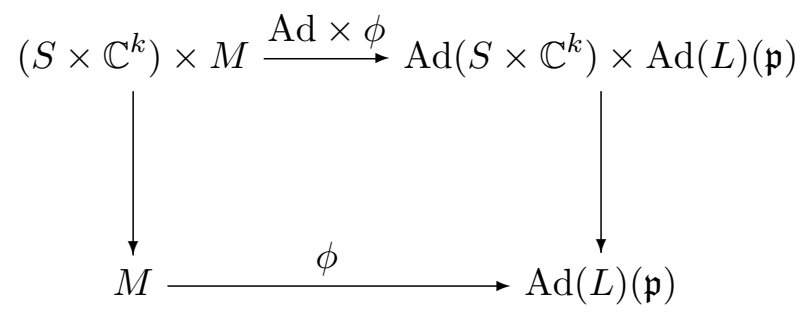

also commutes: we have for $g \in S \times \mathbb{C}^{k}, p=f p_{0} \in M=L \cdot p_{0}$ :

$$
\phi(g p)=\phi\left(g f p_{0}\right)=\operatorname{Ad}(g f) \mathfrak{p}=\operatorname{Ad}(g) \operatorname{Ad}(f) \mathfrak{p}=\operatorname{Ad}(g) \phi(p) .
$$

Let $X$ denote the embedding of $\operatorname{Ad}(L) \mathfrak{p}$ into $\mathbb{P}\left(\Lambda^{k} \mathfrak{l}\right)$. Since the first diagram is also commuting, $X$ is a $S \times \mathbb{C}^{k}$ stable subvariety. Clearly $S \times \mathbb{C}^{k}$ is a solvable 
Lie group and so by the Borel Fixed Point Theorem $S \times \mathbb{C}^{k}$ has a fixed point $p$ in $X$, which of course corresponds to a fixed point of the original action of $S \times \mathbb{C}^{k}$ on $M$. Then it follows

$$
\begin{aligned}
H^{\mathbb{C}} \cdot p & =\left\{g \cdot p \mid g \in H^{\mathbb{C}}\right\} \\
& =\left\{(g s, v) \cdot p \mid(g s, v) \in \tilde{G}_{s} S \times \mathbb{C}^{k}\right\} \\
& =\left\{(g s, a+i b) \cdot p \mid g s \in \tilde{G}_{s} S, a, b \in \mathbb{R}^{k}\right\} \\
& =\left\{((g, a)(s, i b)) \cdot p \mid g s \in \tilde{G}_{s} S, a, b \in \mathbb{R}^{k}\right\} \\
& =\left\{(g, a) \cdot((s, i b) \cdot p) \mid g s \in \tilde{G}_{s} S, a, b \in \mathbb{R}^{k}\right\} \\
& =\left\{(g, a) \cdot p \mid g \in \tilde{G}_{s}, a \in \mathbb{R}^{k}\right\} \\
& =H \cdot p=G \cdot p
\end{aligned}
$$

which proves the theorem. 


\section{Bibliography}

[AlAl 1993] D.V. Alekseevsky and A. Alekseevsky Asystatic G-manifolds, Differential geometry and topology (Alghere, 1992), World Sci. Publishing, River Edge, NJ, (1993), 1-22.

[AlMa 2001] D.V. Alekseevsky and S. Marchiafava, Almost Hermitian and Kähler submanifolds of a quaternionic Kähler manifold, Osaka J. Math. 38 (2001) 869-904.

[AlMa 2005] D.V. Alekseevsky and S. Marchiafava, A twistor construction of Kähler submanifolds of a quaternionic Kähler manifold, Ann. Mat. Pura Appl. 184 (2005), 53-74.

[Ak 1995] D. N. Akhiezer, Lie Group Actions in Complex Analysis, Vieweg \& Sohn Verlagsgesellschaft, Braunschweig/Wiesbaden, 1995.

[At 1982] M. F. Atiyah, Convexity and commuting Hamiltonians, Bull. London Math. Soc. 14 (1982), 1-15.

[Bi 2006] L. Biliotti, Coisotropic and polar actions on compact irreducible Hermitian symmetric spaces, Trans. Amer. Math. Soc. 358 (2006), 3003-3022.

[BiGo 2005] L. Biliotti and A. Gori, Coisotropic and polar actions on complex Grassmannians, Trans. Amer. Math. Soc. 357 (2005), 1731-1751.

[BaNa 1990] W. Barth and R. Narasimhan (Eds.), Several Complex Variables VI, Encyclopaedia of Mathematical Sciences Volume 69, Springer Verlag, Berlin-Heidelberg-New York, 1990.

[Be 1987] A. L. Besse, Einstein Manifolds, vol 10 of Ergebnisse der Mathematik und Grenzgebiete, 3. Folge, Springer, Berlin, Heidelberg, New York, 1987.

[Bo 1991] A. Borel, Linear algebraic groups, second edition, Graduate Texts in Mathematics, No. 126, Springer-Verlag, New York, 1991.

[Bou 1968] Bourbaki, Groups et algèbres de Lie, Chap. 3, Hermann, Paris, 1968.

[BoSa 1958] R. Bott and H. Samelson, Applications of the theory of Morse to symmetric spaces, Amer. J. Math 80 (1958), 964-1029. Correction in Amer. J. Math. 83 (1961), 207-208. 
[Br 1972] G. E. Bredon, Introduction to Compact Transformation Groups, Academic Press, New York and London, 1972.

[Co 1971] L. Conlon, Variational completeness and K-transversal domains, J. Differential Geom. 5 (1971), 135-147.

[Dad 1985] J. Dadok, Polar coordinates induced by actions of compact Lie groups, Trans. Amer. Math. Soc. 288 (1985), 123-137.

[Da 1996] R. Dabrowski, On normality of the closure of a generic torus orbit in $G / P$, Pacific J. of Math 172 (1996), 321-330.

[DuKo 2000] J. J. Duistermaat and J. A. C. Kolk, Lie Groups, Springer Verlag, New York-Heidelberg-Berlin, 2000.

[FlHa 1991] H. Flaschka and L. Haine, Torus orbits in G/P, Pacific. J. Math., 149 (1991), 251-292.

[GeSe 1987] I.M. Gel'fand and V.V. Serganova, Combinatorial geometries and torus strata on homogeneous compact manifolds, Russian Math. surveys 42 (1987), 133-168.

[GoPo 2006] A. Gori and F. Podestà, Symplectically asystatic actions of compact Lie groups, Transform. Groups 11 (2006), 177-184.

[Go 2004] C. Gorodski, Polar actions on compact symmetric spaces which admit a totally geodesic principal orbit, Geometriae Dedicata 103 (2004), 193204.

[Gr 1965] A. Gray, A note on manifolds whose holonomy is a subgroup of $\operatorname{Sp}(n) \cdot \operatorname{Sp}(1)$, Mich. Math. J. 16 (1965).

[GuSt 1982] V. Guillemin and S. Sternberg, Convexity properties of the moment mapping, Invent. Math. 67 (1982), 491-513.

[Gu St 1984] V. Guillemin and S. Sternberg, Symplectic Techniques in Physics, Cambridge University Press, Cambdrige, 1984.

[GuRo 1965] R. C. Gunning and H. Rossi, Analytic Functions in Several Complex Variables, Prentice-Hall, Englewood cliffs, N.J., 1965.

[Ha 1977] R. Hartshorne, Algebraic Geometry, Springer Verlag, New YorkHeidelberg-Berlin, 1977.

[HPTT 1994] E. Heintze, R. S. Palais, C.-L. Terng and G. Thorbergsson, $H y$ perpolar actions and $k$-flat homogeneous spaces, J. reine angew. Math. $\mathbf{4 5 4}$ (1994), 163-179.

[He 1962] S. Helgason, Differential Geometry and Symmetric Spaces, Academic Press, New York and London, 1962.

[Ho 1965] G. Hochschild, The Structure of Lie Groups, Holden-Day, San Francisco, London, Amsterdam, 1965. 
[HuWu 1990] A. T. Huckleberry and T. Wurzbacher, Multiplicity-free complex manifolds Math. Annalen 286 (1990) 261-280.

[Hu 1975] J. E. Humphreys, Linear algebraic groups, Graduate Texts in Mathematics, No. 21, Springer-Verlag, New York-Heidelberg, 1975.

[Ki 1984] F. Kirwan, Convexity properties of the moment mapping III, Invent. Math. 77 (1984), 547-552.

[Kn 1996] A. W. Knapp, Lie Groups Beyond an Introduction, Birkhäuser, Boston, Basel, Berlin, 1996

[Ko 1972] S. Kobayashi, Transformation Groups in Differential Geometry, Springer Verlag, Berlin-Heidelberg-New York, 1972.

[KoNo 1963] S. Kobayashi and K. Nomizu, Foundations of Differential Geometry, vol. I, Interscience Publishers, J. Wiley \& Sons, 1963.

[KoNo 1969] S. Kobayashi and K. Nomizu, Foundations of Differential Geometry, vol. II, Interscience Publishers, J. Wiley \& Sons, 1969.

[Kol 1998] A. Kollross, A classification of hyperpolar and cohomogeneity one actions, $\mathrm{PhD}$ thesis, Augsburg (1998).

[Kol 2006] A. Kollross, Polar actions on symmetric spaces, arXiv:math.DG/0506312, to appear in Journal of Differential Geometry.

[Kos 1955] B. Kostant, Holonomy and the Lie algebra in infinitesimal motions of a Riemannian manifold, Trans. Amer. Math. Soc. 80 (1955), 528-542.

[MSZ 1956] D. Montgomery, H. Samelson and L. Zippin, Singular points of a compact transformation group, Ann. of Math. 63 (1956), 1-9.

[MSY 1956] D. Montgomery, H. Samelson and C. T. Yang, Exceptional orbits of highest dimension, Ann. of Math. 64 (1956), 131-141.

[NiTa 1987] T. Nitta and M. Takeuchi, Contact structures on twistor spaces, J. Math. Soc. Japan 39 (1987) 139-162.

[Oda 1988] T. Oda, Convex bodies and algebraic geometry - an introduction to the theory of toric varieties, Springer-Verlag, Berlin-Heidelberg-New York, 1988.

[Oda 1991] T. Oda, Geometry of toric varieties, Proc. of the Hyderabad conference on algebraic groups, Manoj Prakashan, Madra-India, 1991.

[PaTe 1987] R. S. Palais and C.-L. Terng, A general theory of canonical forms, Trans. Amer. Math. Soc. 300 (1987), 771-789.

[PaTe 1988] R. S. Palais and C.-L. Terng, Critical point theory and submanifold geometry, Lecture Notes in Mathematics 1353, Springer-Verlag, BerlinNewYork, 1988. 
[PoTh 1999] F. Podestà and G. Thorbergsson, Polar actions on rank one symmetric spaces, J. Differential Geom. 53 (1999), 131-175.

[PoTh 2002] F. Podestà and G. Thorbergsson, Polar and coisotropic actions on Kähler manifolds, Trans. Amer. Math. Soc. 354 (2002), 1759-1781.

[PoTh 2003] F. Podestà and T. Thorbergsson, Coisotropic actions on compact homogeneous Kähler manifolds. Math. Z. 243 (2003), 471-490.

[PoSa 1991] Y.S. Poon and M. Salamon, Quaternionic Kähler 8-manifolds with positive scalar curvature, J. Differential Geom. 33 (1991), 363-378.

[Sa 1992] T. Sakai, Riemannian Geometry, AMS Providence, Rhode Island, 1992.

[Sa 1982] S. M. Salamon, Quaternionic Kähler manifolds, Invent. Math 67 (1982), 143-171.

[Sa 1999] S. M. Salamon, Quaternion Kähler geometry, Surv. Differ. Geom. VI, Int. Press, Boston, MA, (1999), 83-121

[SCM 1997] C. U. Sánchez, A. L. Calí and J. L. Moreschi, Spheres in Hermitian symmetric spaces and flag manifolds, Geom. Dedicata 64 (1997), 261-276.

[Va 1984] V.S. Varadarajan, Lie Groups, Lie Algebras, and Their Representations, Springer Verlag, New York-Berlin-Heidelberg-Tokyo, 1984.

[Wo 1965] J. A. Wolf, Complex homogeneous contact manifolds and quaternionic symmetric spaces, J. Math. Mech., 14 (1965), 1033-1047. 
Ich versichere, dass ich die von mir vorgelegte Dissertation selbständig angefertigt, die benutzten Quellen und Hilfsmittel vollständig angegeben und die Stellen der Arbeit - einschließlich Tabellen, Karten und Abbildungen -, die anderen Werken im Wortlaut oder dem Sinn nach entnommen sind, in jedem Einzelfall als Entlehnung kenntlich gemacht habe; dass diese Dissertation noch keiner anderen Fakultät oder Universität zur Prüfung vorgelegen hat; dass sie abgesehen von unten angegebenen Teilpublikationen - noch nicht veröffentlicht worden ist sowie dass ich eine solche Veröffentlichung vor Abschluss des Promotionsverfahrens nicht vornehmen werde.

Die Bestimmungen der Promotionsordnung sind mir bekannt. Die von mir vorgelegte Dissertation ist von Herrn Prof. Dr. Gudlaugur Thorbergsson betreut worden.

Teilpublikationen: keine 


\section{Lebenslauf}

Name

Samuel Tebege

Geburtsdatum 14.06.1979

Geburtsort Addis Abeba, Äthiopien

\section{Schulbildung}

1986-1990

Grundschule Merian Str., Köln

1990-1999

Friedrich-Wilhelm-Gymnasium, Köln

Abschluss mit Abitur

\section{Studium}

10.1999-12.2003

Studium der Mathematik (Diplom) mit Nebenfach

Informatik an der Universität zu Köln

10.2001

Vordiplom

12.2003

Diplom

Thema der Diplomarbeit: Polar and coisotropic actions on Kähler manifolds

seit 1.2004

Promotionsstudium der Mathematik an der Universität zu Köln

\section{Berufstätigkeit}

10.2001-12.2003 Studentische Hilfskraft am Mathematischen Institut der Universität zu Köln

seit 01.2004

Wissenschaftlicher Mitarbeiter am Mathematischen Institut der Universität zu Köln 